\title{
Global Isochrons and Phase Sensitivity of Bursting Neurons*
}

\author{
Alexandre Mauroy ${ }^{\dagger}$, Blane Rhoads ${ }^{\dagger}$, Jeff Moehlis ${ }^{\dagger}$, and Igor Mezić ${ }^{\dagger}$
}

Abstract. Phase sensitivity analysis is a powerful method for studying (asymptotically periodic) bursting neuron models. One popular way of capturing phase sensitivity is through the computation of isochrons - subsets of the state space that each converge to the same trajectory on the limit cycle. However, the computation of isochrons is notoriously difficult, especially for bursting neuron models. In [W. E. Sherwood and J. Guckenheimer, SIAM J. Appl. Dyn. Syst., 9 (2010), pp. 659-703], the phase sensitivity of the bursting Hindmarsh-Rose model is studied through the use of singular perturbation theory: cross sections of the isochrons of the full system are approximated by those of fast subsystems. In this paper, we complement the previous study, providing a detailed phase sensitivity analysis of the full (three-dimensional) system, including computations of the full (twodimensional) isochrons. To our knowledge, this is the first such computation for a bursting neuron model. This was made possible thanks to the numerical method recently proposed in [A. Mauroy and I. Mezić, Chaos, 22 (2012), 033112] -relying on the spectral properties of the so-called Koopman operator - which is complemented with the use of adaptive quadtree and octree grids. The main result of the paper is to highlight the existence of a region of high phase sensitivity called the almost phaseless set and to completely characterize its geometry. In particular, our study reveals the existence of a subset of the almost phaseless set that is not predicted by singular perturbation theory (i.e., by the isochrons of fast subsystems). We also discuss how the almost phaseless set is related to empirically observed phenomena such as addition/deletion of spikes and to extrema of the phase response of the system. Finally, through the same numerical method, we show that an elliptic bursting model is characterized by a very high phase sensitivity and other remarkable properties.

Key words. isochrons, bursting, phase response, Koopman operator

AMS subject classifications. $65 \mathrm{P} 99,37 \mathrm{~N} 25,70 \mathrm{~K} 70,37 \mathrm{C} 10$

DOI. $10.1137 / 130931151$

1. Introduction. Bursting is a behavior commonly observed in neurons. Whereas tonic firing is characterized by isolated spikes, bursting is characterized by the alternation between a rapid succession of spikes (i.e., the burst) and a quiescent state. This behavior is involved in many physiological mechanisms, including the generation of respiratory rhythms in the preBötzinger complex [5], the transmission of signals from the hypothalamus (e.g., in the brain reward circuit [9]), and even the secretion of insulin in pancreatic $\beta$-cells [8]. Abnormal bursting activity has also been reported in the basal ganglia [21,39] in the context of Parkinson's disease.

\footnotetext{
${ }^{*}$ Received by the editors July 30, 2013; accepted for publication (in revised form) by J. Guckenheimer November 19, 2013; published electronically March 4, 2014. This research was funded by Army Research Office grant W911NF11-1-0511 with Program Manager Dr. Sam Stanton.

http://www.siam.org/journals/siads/13-1/93115.html

${ }^{\dagger}$ Department of Mechanical Engineering, University of California Santa Barbara, Santa Barbara, CA 93106 (alex. mauroy@engr.ucsb.edu, blanerhoads@engr.ucsb.edu, moehlis@engineering.ucsb.edu, mezic@engineering.ucsb.edu). Part of this work was completed while A. Mauroy held a postdoctoral fellowship from the Belgian American Educational Foundation.
} 
Mathematical bursting neuron models exhibit complex multidimensional dynamics. However, in many cases, they admit a limit cycle, and the dynamics can be reduced to onedimensional phase dynamics $[30,46]$. This phase reduction in turn leads to the concept of phase sensitivity: how the neuron responds to a given external stimulus at a given state. The phase reduction framework is extremely important for investigating the dynamics of neuronal populations in a general context (e.g., [3, 12, 27, 29]) and might also be useful for the more recent study of synchronization of bursting neurons [26].

Phase reduction is obtained through the partition of the basin of attraction into so-called isochrons - sets of points converging to the same trajectory on the limit cycle and therefore sharing the same (asymptotic) phase [47]. In other words, the isochrons are interpreted as the level sets of the phase function. Since the isochrons are directly related to the phase dynamics of the system, they provide a global picture of the (potentially very complex) dynamics.

The computation and visualization of isochrons is much easier if confined to the immediate vicinity of the limit cycle, particularly for slow-fast dynamics. But, especially in the case of (bursting) neurons, the computation of the isochrons in the whole basin of attraction-i.e., the global isochrons - is desirable not only because neurons can be perturbed by large stimuli but also because a local approximation in the vicinity of the limit cycle does not usually capture the full complex geometry of the isochrons. In [34] the global isochrons were computed for a two-dimensional reduced Hodgkin-Huxley model via a two point boundary value continuation method. (Other recent methods can also be found in [20, 24].) As stated by the authors, a higher-dimensional version of this method, although theoretically straightforward, would present challenges numerically and has not yet been implemented. In addition to their three (or higher) dimensions, bursting neuron models have particularly complex limit cycles and dynamics, making the computation of isochrons even harder. A preliminary solution to this problem was given in [41]. In that work, the authors used singular perturbation theory, approximating the isochrons of the bursting Hindmarsh-Rose model by the (one-dimensional) isochrons of two-dimensional fast subsystems. However, they suggested that a direct computation of the (two-dimensional) isochrons for the full model is necessary for a complete understanding of the system dynamics.

In this paper, we compute the two-dimensional isochrons of the full Hindmarsh-Rose bursting neuron model, thereby providing a complete phase sensitivity description of the model. The results are obtained with the numerical method recently proposed in [31], which computes the isochrons as the level sets of Fourier averages evaluated along the system trajectories, utilizing the Koopman operator framework for dynamical systems analysis [4, 32]. This method is complemented with the use of adaptive grids (quadtree- and octree-based), which enable better detail in regions where the isochrons are more dense.

Our results show that the complex geometry of the isochrons is not accurately captured by the fast subsystems, confirming the need to compute the isochrons for the full system. In particular, the results reveal the existence of an almost phaseless set - a region of particularly high concentration of isochrons extending throughout the state space. Motivating the use of adaptive grids, the almost phaseless set is the focus of our attention, since it explains various phenomena that have been empirically observed in earlier studies. For instance, the early onset or termination of the burst - and more generally the addition or deletion of spikesunder particular perturbations is directly interpreted as the crossing of the almost phaseless 
set. Moreover, computations of (noninfinitesimal) phase responses reveal the proximity of the almost phaseless set to the limit cycle and suggest efficient strategies for triggering large phase shifts.

In order to complement the study of the Hindmarsh-Rose model, which exhibits exclusively square-wave bursting, we briefly investigate the phase sensitivity of an elliptic bursting model. In this case, we observe that the almost phaseless set fills the state space much more densely than in the Hindmarsh-Rose model. Furthermore, zooming in on a one-dimensional cross section reveals a surprising fractal pattern of smaller and smaller features. This indicates a much more extreme and irregular overall phase sensitivity and makes the full twodimensional isochrons too complex to be computed, even with the present adaptive grid refinement.

The paper is organized as follows. In section 2, we introduce the mathematical bursting neuron models and the notions of phase response and isochrons. In addition, we motivate our study by comparing the isochrons of one of the fast subsystems of the Hindmarsh-Rose model with the corresponding cross section of the isochrons of the full system. Section 3 presents the numerical methods, i.e., the Fourier average method and the adaptive (quadtree and octree) grids. In section 4, the isochrons of the full Hindmarsh-Rose model are described in the three-dimensional state space but also in cross sections of interest. Section 5 describes the phase response for noninfinitesimal impulsive perturbations. The case of elliptic bursting is considered in section 6 . Concluding remarks are given in section 7 .

\section{Preliminaries.}

2.1. Bursting neuron models. The membrane voltage of bursting neuron models alternates between a quiescent state and an active bursting state, where one observes a rapid succession of spikes. This behavior is due to the interplay between several fast variables (including the membrane voltage and fast ion current(s)) and one (or two in some cases such as parabolic bursting) slow recovery variable(s).

The bursting mechanism can be better understood through the so-called dissection of the bursting [38]. In this approach the system is decomposed into a family of fast subsystems parametrized by the slow variable, which acts as a bifurcation parameter. Each fast subsystem admits a stable limit cycle and/or a stable fixed point. The family of limit cycles parametrized by the slow variable is denoted by $\tilde{\Gamma}_{s}$ and corresponds to the spikes in the bursting segment of the full system. The family of stable fixed points parametrized by the slow variable is denoted by $\tilde{Q}_{s}$ and is related to the quiescent state of the full system. Through the bifurcations that occur as the slow variable is modified, destruction of the limit cycle or fixed point leads to termination of the active bursting or quiescent state, respectively. Models can be classified according to the bifurcations involved in the bursting mechanism $[25,36]$. In this context, unfolding theory provides a useful framework for classifying bursting models $[1,16]$ and for unifying them $[15,35]$.

The first model considered in this paper is the Hindmarsh-Rose (HR) model [22], which is one of the most popular bursting neuron models [42]. It is defined by the three-dimensional dynamics

$$
\dot{V}=n-a V^{3}+b V^{2}-h+I,
$$




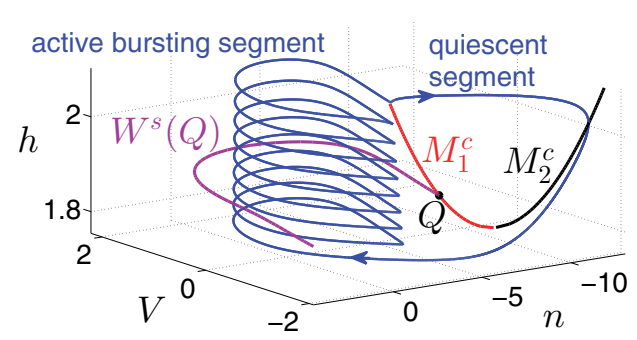

(a)

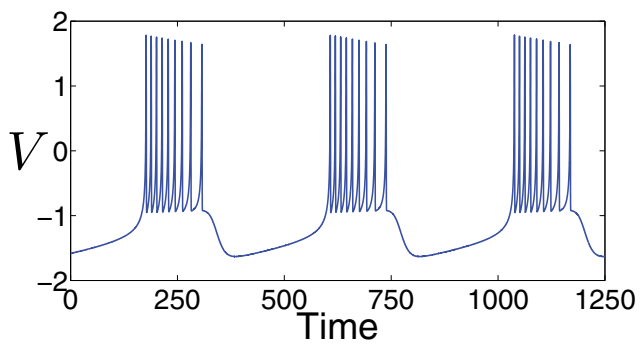

(b)

Figure 1. The HR model exhibits periodic (square-wave) bursting behavior. (a) Limit cycle in the state space, with the stable manifold $W^{s}(Q)$ of the saddle point $Q$ and the critical manifolds $M_{1}^{c}$ and $M_{2}^{c}$. (b) Periodic evolution of the membrane voltage $V$.

$$
\begin{aligned}
\dot{n} & =c-d V^{2}-n, \\
\dot{h} & =r\left(\sigma\left(V-V_{0}\right)-h\right),
\end{aligned}
$$

where $V$ is the membrane voltage and $n$ and $h$ are the ion currents. If $r \ll 1$, then $h$ is a slow recovery variable and parameterizes the family of fast subsystems given by (2.1)-(2.2). Throughout the manuscript, we adopt the usual parameters $a=1, b=3, c=1, d=5$, $r=0.001, \sigma=4, V_{0}=-1.6, I=2$. With these parameters, the full system admits a stable limit cycle of period $T_{0} \approx 430.786$ characterized by an active bursting segment (with nine spikes) and by a quiescent segment (Figure 1(a)). The system also admits a saddle point $Q$, at $(V, h, n) \approx(1.127,-5.353,1.891)$, with a one-dimensional stable manifold $W^{s}(Q)$ (magenta curve in Figure 1), and two one-dimensional slow manifolds $S_{1}$ and $S_{2}$ (not in the figure). The slow manifold $S_{1}$ lies close to the critical manifold $M_{1}^{c}$ (red curve in Figure 1), which corresponds to the family of saddle points of the fast subsystems (denoted by $\tilde{Q}_{s}$ in Figure 2 (a) below). The slow manifold $S_{2}$ lies close to the critical manifold $M_{2}^{c}$ (black curve in Figure 1 ), which corresponds to the family of stable fixed points $\tilde{Q}_{s}$ of the fast subsystems. (See e.g., [22, 41, 42] for more details on the HR model and its bursting dynamics.)

The HR model exhibits square-wave bursting (i.e., type I bursting): the stable limit cycle $\tilde{\Gamma}_{s}$ of the fast subsystem disappears through a homoclinic bifurcation, while the stable fixed point $\tilde{Q}_{s}$ disappears through a saddle-node bifurcation. The termination of the burst (a homoclinic bifurcation) occurs when $M_{1}^{c}$ intersects the bursting segment of the limit cycle, at $h \approx 2.09$. The orbit of the limit cycle then enters the quiescent state, following $M_{2}^{c}$ until it merges with $M_{1}^{c}$ (in a saddle-node bifurcation) at $h \approx 1.8$.

2.2. Phase reduction: Isochrons and phase response. Phase reduction is a powerful method for studying complex asymptotically periodic dynamics, such as the HR dynamics (2.1)-(2.3). In this framework, the multidimensional state dynamics are reduced to onedimensional phase dynamics, thanks to the important concepts of isochrons and phase response. In the case of bursting neurons, the isochrons and the phase response provide direct information on how the bursting behavior is modified under the effect of particular external stimuli. In this section, we briefly review the framework in a general context which is not limited to bursting neuron models. 
Consider a dynamical system,

$$
\dot{\mathbf{x}}=\mathbf{F}(\mathbf{x}), \quad \mathbf{x} \in \mathbb{R}^{n},
$$

which admits a stable limit cycle $\Gamma$ of period $T_{0}$, with a basin of attraction $\mathcal{B}(\Gamma) \subseteq \mathbb{R}^{n}$. We denote by $\phi: \mathbb{R}^{+} \times \mathbb{R}^{n} \rightarrow \mathbb{R}^{n}$ the flow induced by (2.4); that is, $\phi\left(t, \mathbf{x}_{0}\right)$ is the solution of (2.4) for the initial condition $\mathbf{x}(0)=\mathbf{x}_{0}$. According to [46], a phase $\theta=2 \pi t / T_{0} \in \mathbb{S}^{1}$, where $t \in\left[0, T_{0}\right)$, is assigned to the point $\mathbf{x}^{\gamma} \in \Gamma$ that satisfies $\mathbf{x}^{\gamma}=\phi\left(t, \mathbf{x}_{0}^{\gamma}\right)$. (The point $\mathbf{x}_{0}^{\gamma}$, which corresponds to the phase $\theta=0$, is arbitrarily chosen on the limit cycle.) It follows that the phase dynamics on the limit cycle are $\dot{\theta}=\omega_{0}, \omega_{0} \triangleq 2 \pi / T_{0}$.

Isochrons. The notion of phase is extended to the whole basin of attraction $\mathcal{B}(\Gamma)$ through the concept of isochrons [47]. The isochron $\mathcal{I}_{\theta}$ is the set of states that converge to the limit cycle trajectory associated with the phase $\theta$. Rigorously, it is defined as the $(n-1)$-dimensional manifold

$$
\mathcal{I}_{\theta}=\left\{\mathbf{x} \in \mathcal{B}(\Gamma) \mid \lim _{t \rightarrow \infty}\left\|\phi(t, \mathbf{x})-\phi\left(t+\theta / \omega_{0}, \mathbf{x}_{0}^{\gamma}\right)\right\|=0\right\} .
$$

The isochrons extend the phase dynamics $\dot{\theta}=\omega_{0}$ to the entire basin of attraction and allow the definition of the phase function $\Theta: \mathcal{B}(\Gamma) \rightarrow \mathbb{S}^{1}$ as that which satisfies

$$
\Theta(\mathbf{x})=\theta \Longleftrightarrow \mathbf{x} \in \mathcal{I}_{\theta} \text {. }
$$

The isochrons are the invariant fibers of the stable manifold of the limit cycle, i.e., of the basin of attraction $\mathcal{B}(\Gamma)$. They are invariant under the time- $T$ map $\phi(T, \cdot)$ and, more precisely, satisfy (with a slight abuse of notation) $\phi\left(t, \mathcal{I}_{\theta}\right)=\mathcal{I}_{\theta+\omega_{0} t}$. Since the isochrons partition the basin of attraction into sets that are equally spaced in time, they provide a global picture of the (possibly complex) dynamics of the system.

In addition, the isochrons fully capture the phase sensitivity of the dynamics to external perturbations. For instance, suppose that a trajectory on the limit cycle is subjected to an instantaneous perturbation or input $\mathbf{u}(t)=\boldsymbol{\Delta} \mathbf{x} \delta(t)$, where $\delta(t)$ is the Dirac function, such that the trajectory jumps from one state $\mathbf{x}^{\gamma} \in \Gamma$ in some isochron $\mathcal{I}_{\theta}$ to another state $\mathbf{x}^{\gamma}+\boldsymbol{\Delta} \mathbf{x}$ in some different isochron $\mathcal{I}_{\theta+\Delta \theta}$. In other words, the trajectory undergoes a phase shift $\Delta \theta=\Theta\left(\mathbf{x}^{\gamma}+\Delta \mathbf{x}\right)-\Theta\left(\mathbf{x}^{\gamma}\right)$, which advances (by $\left.\Delta \theta \in(0, \pi]\right)$ or delays (by $\Delta \theta \in[-\pi, 0)$ ) its asymptotic behavior. The size of this phase shift is determined by (a) the general phase sensitivity or gradient, as indicated by the concentration of isochrons, and (b) the direction of the isochrons relative to the direction of the perturbation $\Delta \mathbf{x}$.

Phase response. Phase sensitivity can be evaluated along the limit cycle, leading to the concept of phase response. The most common such notion is that of the infinitesimal phase response curve (iPRC) [11,27], which gives the phase sensitivity to an infinitesimal perturbation in a given unit direction e. Note that in the case of a neuron model, the direction e typically corresponds to the membrane voltage state (i.e., usually $(1,0, \ldots, 0))$. More rigorously, the iPRC is defined as the function $Z_{i}: \mathbb{S}^{1} \rightarrow \mathbb{R}$ that satisfies

$$
Z_{i}(\theta)=\left\langle\nabla \Theta\left(\mathbf{x}^{\gamma}(\theta)\right), \mathbf{e}\right\rangle,
$$

where $\langle\cdot, \cdot\rangle$ denotes the inner product and $\mathbf{x}^{\gamma}=\mathcal{I}_{\theta} \cap \Gamma$. Since the iPRC depends only on the local dynamics in the neighborhood of the limit cycle, it can be computed through efficient numerical methods (e.g., [17]). 
For weak inputs $u(t) \ll 1$, the iPRC provides a good approximation of the state dynamics $\dot{\mathbf{x}}=\mathbf{F}(\mathbf{x})+u(t) \mathbf{e}$ in terms of the phase dynamics

$$
\dot{\theta}=\omega_{0}+Z_{i}(\theta) u(t)
$$

(See, e.g., [3], for more detail.) In contrast, for stronger perturbations, which push the trajectories farther from the limit cycle, the iPRC does not provide a good approximation of the phase sensitivity, since it retains only local information on the limit cycle dynamics. In this context, global phase responses must be considered instead. In [20], the quantity $\langle\nabla \Theta(\mathbf{x}), \mathbf{e}\rangle$, called the phase response surface, was proposed as a straightforward generalization of the iPRC to the entire basin of attraction. However, the phase difference induced by a finite perturbation $\Delta \mathbf{x}=\alpha \mathbf{e}$ is not directly provided by the phase response surface but by its integral along the direction e. This leads to what we will call the phase response function (PRF), $Z: \mathbb{S}^{1} \times \mathbb{R} \rightarrow \mathbb{S}^{1}$, defined by

$$
Z(\theta, \alpha)=\Theta\left(\mathbf{x}^{\gamma}(\theta)+\alpha \mathbf{e}\right)-\Theta\left(\mathbf{x}^{\gamma}(\theta)\right)=\Theta\left(\mathbf{x}^{\gamma}(\theta)+\alpha \mathbf{e}\right)-\theta
$$

In this paper, we will focus on the PRF rather than on the phase response surface, since, away from the limit cycle, we are more interested in the net effect of a noninfinitesimal perturbation than the local phase sensitivity.

Finally, along these same lines, we are also interested in the "efficiency" of perturbations, as described, for $\alpha \neq 0$, by the ratio

$$
\frac{Z(\theta, \alpha)}{\alpha}
$$

Removing the singularity at $\alpha=0$ yields what we will call the phase response efficiency (PRE), defined by

$$
Z_{e}(\theta, \alpha)=\lim _{\beta \rightarrow 0} \frac{Z(\theta, \alpha+\beta)}{\alpha+\beta}
$$

Note that this is the average (rather than integral) along the direction e of the phase response surface $\langle\nabla \Theta(\mathbf{x}), \mathbf{e}\rangle$. Also, the PRE, phase response surface, and iPRC are all equivalent along $\alpha=0$, since

$$
Z_{e}(\theta, 0)=\lim _{\beta \rightarrow 0} \frac{Z(\theta, \beta)}{\beta}=\lim _{\beta \rightarrow 0} \frac{\Theta\left(\mathbf{x}^{\gamma}(\theta)+\beta \mathbf{e}\right)-\theta}{\beta}=\left\langle\nabla \Theta\left(\mathbf{x}^{\gamma}(\theta)\right), \mathbf{e}\right\rangle=Z_{i}(\theta) .
$$

In this sense the $Z_{e}$ surface may be thought of as a continuum of generalized phase response curves.

As shown in section 2.3, bursting neuron models have high concentrations of isochrons and large phase responses at small (but finite) distances from the limit cycle. Thus the (finite) $\operatorname{PRF} Z$ and $\operatorname{PRE} Z_{e}$ are of particular interest and will be computed in section 5 . More broadly, the computation of the global isochrons and phase function itself in the entire basin of attraction is of paramount importance. 


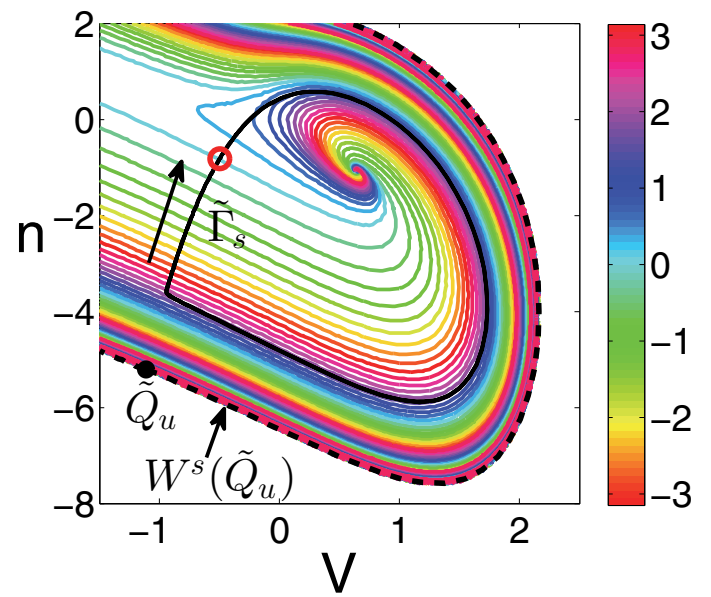

(a)

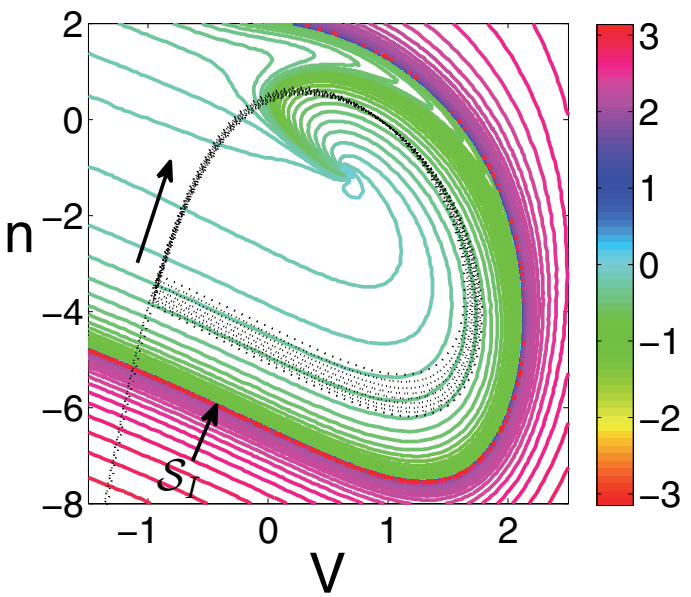

(b)

Figure 2. The isochrons of the fast subsystem for $h=1.9$ (a) are compared with the corresponding slice of the isochrons of the full system (b). The concentration of isochrons observed in (a) near the stable manifold $W^{s}\left(\tilde{Q}_{u}\right)$ of the saddle point $\tilde{Q}_{u}$ is recovered in (b) as an almost phaseless set $\mathcal{S}_{I}$. (The isochrons are obtained with the methods described in section 3. In (a), the isochrons are spaced in phase by $\pi / 10$. The red circle represents the point of the limit cycle associated with a phase $\theta=0$. In $(\mathrm{b})$, the isochrons are spaced in phase by $\pi / 50$. The black dashed curve is the projection of the limit cycle onto the cross section.)

2.3. Isochrons of fast subsystems versus isochrons of the full system. According to singular perturbation theory $[13,14]$, the geometry of the isochrons of bursting models can be approximated by the isochrons of the fast subsystems. For instance, the isochrons of the HR model were studied in [41] through the isochrons of the fast subsystems (2.1)-(2.2). However, we stress here that this approach does not capture the complete geometry of the isochrons and therefore cannot be used for a comprehensive and accurate phase sensitivity analysis of the model.

Figure 2 shows (a) the one-dimensional isochrons of the fast subsystem (2.1)-(2.2) parametrized by $h=1.9$ and (b) the $h=1.9$ cross section of the two-dimensional isochrons of the full system, computed using the numerical methods of section 3. First, it is important to note that these two families of isochrons are associated with two different limit cycles (with different periods) and are not related to the same phase $\theta$. For instance, the complete set of isochrons (over the range $[-\pi, \pi)$ ) is observed in the fast subsystem, while the isochrons do not all appear in the section $h=1.9$ of the full system. Also, the point of the limit cycle associated with the phase $\theta=0$ is as represented by a red circle for the fast subsystem. In contrast, the isochron $\theta=0$ does not intersect the limit cycle in the section $h=1.9$ for the full system. In the fast subsystem, the stable manifold $W^{s}\left(\tilde{Q}_{u}\right)$ of the saddle point $\tilde{Q}_{u}$ (black dashed curve) is the separatrix between the basins of attraction of the limit cycle $\tilde{\Gamma}_{s}$ and the stable fixed point $\tilde{Q}_{s}$ near $(-10.7,-1.5)$ (not included in the picture). Therefore, according to $[18,47], W^{s}\left(\tilde{Q}_{u}\right)$ is a phaseless set, and every isochron comes arbitrarily close to it. A similar observation is obtained with the full system. In this case, the (two-dimensional) stable manifold $W^{s}\left(S_{1}\right)$ of the slow manifold $S_{1}$ —computed, for instance, in [19] for a similar bursting model—surrounds 


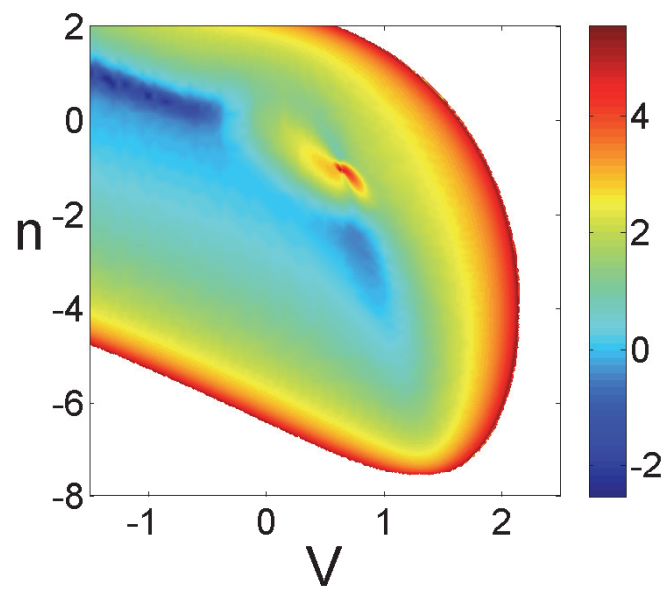

(a)

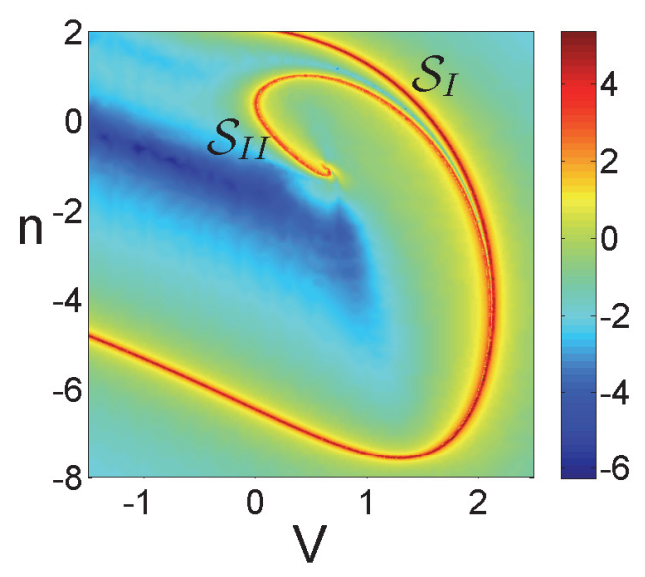

(b)

Figure 3. The gradient of the phase function $\nabla \Theta$ (shown as $\ln \|\nabla \Theta\|$ ) is computed in the fast subsystem for $h=1.9$ (a) and in the corresponding slice for the full system (b). The full system exhibits a concentration of isochrons inside the bursting segment (i.e., $\mathcal{S}_{I I}$ ) that is not observed with the fast subsystem.

the bursting segment of the limit cycle and approximately corresponds to the set of stable manifolds $W^{s}\left(\tilde{Q}_{u}\right)$ in the fast subsystems. The stable manifold $W^{s}\left(S_{1}\right)$ therefore also plays the role of separatrix between two "basins of attraction" of the limit cycle, separating the trajectories that initially converge to the bursting segment of the limit cycle and those that initially converge to the quiescent segment of the limit cycle. Along $W^{s}\left(S_{1}\right)$, we observe a concentration of isochrons, similar to the concentration observed with the fast subsystems. However, this does not correspond to a phaseless set, but to an almost phaseless set (a term coined in [34]), since it lies in the interior of the basin of attraction of the limit cycle. (The phaseless set is actually $W^{s}(Q)$; see section 4.1.) Also, the isochrons come exponentially close (rather than arbitrarily close) to this almost phaseless set.

Even though the isochrons of the fast subsystems and the full system are similar, they are also characterized by important differences. In particular, Figure 2(b) exhibits a concentration of isochrons inside the bursting segment of the limit cycle, while nothing is observed in Figure 2(a). This difference is also clearly illustrated in Figure 3, which shows the magnitude of the gradient of the phase function. The concentration of isochrons lying inside the bursting segment is another part of the almost phaseless set, which we denote by $\mathcal{S}_{I I}$, as opposed to $\mathcal{S}_{I}=W^{s}\left(S_{1}\right)$ described earlier. The set $\mathcal{S}_{I I}$ can be interpreted as a separatrix between two "spike regions," that is, two small basins of attraction associated with a spike of the bursting active segment (see section 4.2.1). In contrast to $\mathcal{S}_{I}, \mathcal{S}_{I I}$ involves the dynamics of the slow variable $h$. This second part of the almost phaseless set is therefore not predicted through singular perturbation theory and cannot be captured by the fast subsystems.

Remark 1. Singular perturbation theory cannot be used in a rigorous way to approximate the isochrons of the full system with those of fast subsystems. In the singular limit, the isochrons of the fast subsystems are the fibers of the invariant cylinder obtained by gluing all the limit cycles 
$\tilde{\Gamma}_{s}$ (for $h \in[-\infty, 2.09]$ ). This invariant cylinder is not normally hyperbolic (at the homoclinic bifurcation for $h \approx 2.09$ ) and does not persist in the full system as an invariant manifold. Therefore, the isochrons of the fast subsystems cannot be used to rigorously approximate the fibers of the perturbed cylinder (each of which should be contained in a single isochron of the full system), since such fibers are simply not well-defined. In addition, there is no guarantee that the $h=$ constant cross section of an isochron $\mathcal{I}$ of the full system will be close to an isochron $\tilde{\mathcal{I}}$ of the fast subsystem, even if a fiber contained in $\mathcal{I}$ is close to $\tilde{\mathcal{I}}$. This explains the difference between Figure 2(a) and Figure 2(b).

The entire almost phaseless set $\mathcal{S}=\mathcal{S}_{I} \cup \mathcal{S}_{I I}$ is associated with a high concentration of isochrons and represents a region of high phase sensitivity. For a comprehensive phase sensitivity analysis of the HR bursting model, it is therefore necessary to capture all the features of the almost phaseless set. This requires the computation of the isochrons of the full three-dimensional model.

3. Numerical methods. Computing isochrons is an inherently involved problem. The present method is based on (a) evaluation of Fourier averages along independently computed, forward-in-time trajectories to obtain the phase function $\Theta$ on a grid in the $n$-dimensional state space, and (b) definition of isochrons as level sets of $\Theta$. One of the key advantages of this method is that it allows independent computation of lower-dimensional cross sections of the isochrons. As shown in section 2.3, this has proven useful, for instance, in identifying the fundamental differences that exist between the full HR model and the fast subsystems.

The main challenge of our approach is the large number of computations required to resolve $\Theta$ in areas of highly concentrated isochrons (i.e., the almost phaseless set). To make this more efficient we have implemented the method on adaptive (graded quadtree and octree) grids.

3.1. Fourier averages. The idea of computing the phase function $\Theta$ and isochrons of a spatially complex dynamical system via the method of Fourier averages was first proposed in [31]. In this framework, the function $\Theta$ is related to an eigenfunction of the so-called Koopman operator [32]. For an arbitrary function $f \in C^{1}: \mathbb{R}^{n} \rightarrow \mathbb{R}$ (also called an observable) the Fourier average is defined by

$$
f^{*}(\mathbf{x})=\lim _{T \rightarrow \infty} \frac{1}{T} \int_{0}^{T} f \circ \phi(t, \mathbf{x}) e^{-i \omega_{0} t} d t .
$$

As long as the first Fourier coefficient of the periodic function $f \circ \phi\left(t, \mathbf{x}^{\gamma}\right)$ (where $\mathbf{x}^{\gamma} \in \Gamma$ ) is nonzero, the Fourier average $f^{*}(\mathbf{x})$ is an eigenfunction of the Koopman operator, and its argument gives the desired phase function, that is, $\angle f^{*}(\mathbf{x})=\Theta(\mathbf{x})$ (with the phase $\theta=0$ being determined by the specific choice of $f$ ).

It follows that the isochrons are the level sets of $\angle f^{*}(\mathbf{x})$, and that the PRF satisfies $Z(\theta, \alpha)=\angle f^{*}\left(\mathbf{x}^{\gamma}+\alpha \mathbf{e}\right)-\angle f^{*}\left(\mathbf{x}^{\gamma}\right)$. Although this paper considers only impulsive perturbations, it is important to note that the phase response due to a continuous perturbation $(u(t) \neq \delta(t))$ can also be computed within the framework of Fourier averages. One simply computes the difference of the Fourier average $f^{*}\left(\mathbf{x}^{\gamma}\right)$ between the perturbed and unperturbed trajectories. 


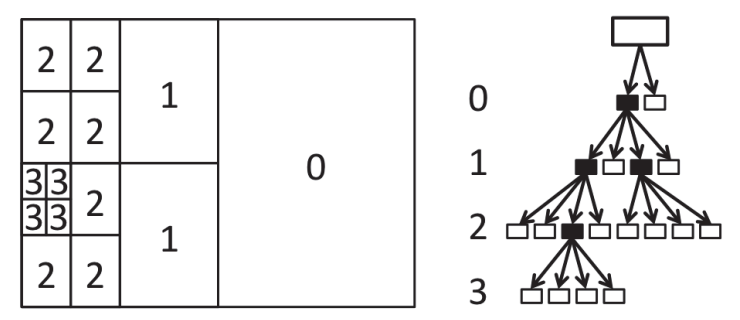

Figure 4. Schematic of graded quadtree grid and tree data structure, with three levels of refinement and two trees corresponding to two initial grid cells in the $x$ direction. Refined and leaf nodes are indicated by filled and unfilled rectangles, respectively, in the right half of the figure.

When the Fourier averages are computed over a finite time horizon $T<\infty$, they are characterized by an error which decreases with the rate $T^{-1}$ [31]. This approximation error can be reduced if one integrates (3.1) over an exact number of $N$ periods $T_{0}$ and disregards an initial time interval $\left[0, T-N T_{0}\right]$, which is related to the transient behavior of the trajectories as they converge toward the limit cycle. This yields the truncated Fourier average

$$
\bar{f}^{*}(\mathbf{x})=\frac{1}{N T_{0}} \int_{T-N T_{0}}^{T} f \circ \phi(t, \mathbf{x}) e^{-i \omega_{0} t} d t
$$

for which the integration time $T$ can be chosen at a reasonably small value (typically a small multiple of the natural period of the limit cycle). See Appendix B for the chosen simulation parameters, including the choice of the function $f$.

3.2. Adaptive grids. The use of graded quadtree and octree adaptive grids has proved crucial for making the present method efficient and accurate. For our purposes, a quadtree (resp. octree) is a tree data structure whose nodes correspond to rectangular grid cells of the two-dimensional (resp., three-dimensional) state space domain. Each node points to either four (resp., eight) "child" nodes - if the corresponding grid cell has been "refined" - or none in which case it is a "leaf" node. Each grid cell is characterized by its "level" (of refinement) in the overall tree, which is related to its size. All of the quadtree and octree grids are graded in the sense that the levels of two (nondiagonally) neighboring cells may not differ by more than one. A tree with maximum level $n$ has exactly one "root" node (i.e., level 0) and at most $4^{n}$ (resp., $8^{n}$ ) leaf nodes. Note that the initial grid usually contains several cells, each of which corresponds to the root node of an individual tree. The number of these initial cells and the overall size of the grid determine the aspect ratio of all cells that the grid will ever contain.

An example of a simple graded quadtree grid and a schematic of the tree data structure are shown in Figure 4. If, for instance, one needed to refine the top-right level 2 cell, here one would also have to refine the top level 1 cell and thus, by recursion, the level 0 cell to maintain the gradedness of the grid.

The grid refinement process consists of the following steps:

1. compute the phase at each new vertex and cell center, via the method of section 3.1; 


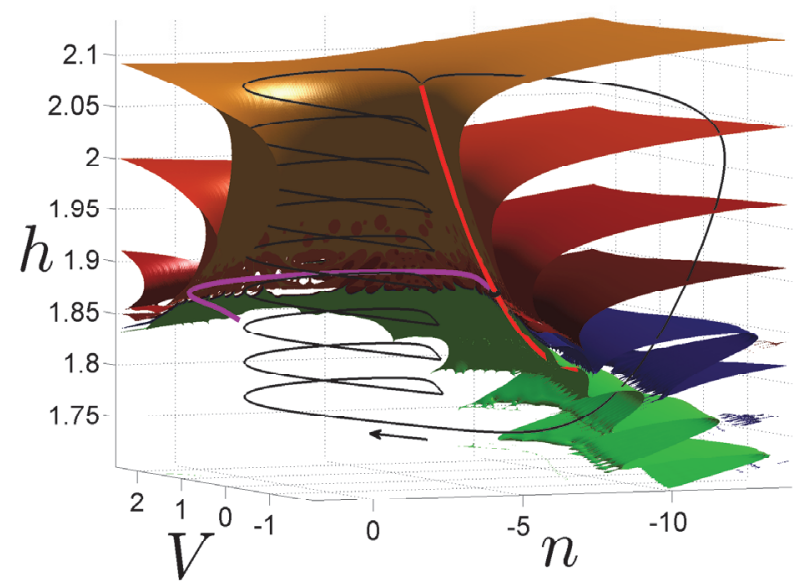

Figure 5. The isochrons $\theta \in\{3 \pi / 6,4 \pi / 6,5 \pi / 6, \pi,-5 \pi / 6,-4 \pi / 6\}$ (from top to bottom) that lie in the quiescent segment are parallel to the $V-n$ plane and surround the active bursting segment. At the end of the quiescent segment, the geometry of the isochrons becomes more complex and cannot be captured completely with the current level of refinement (see Figure 16). The magenta curve is the stable manifold $W^{s}(Q)$ (i.e., phaseless set), and the red curve is the critical manifold $M_{1}^{c}$. Note that the presence of holes near $W^{s}(Q)$ is inherent to the computation of dense level sets for a function with values on $\mathbb{S}^{1}$ (see the discussion in Appendix A.3).

2. determine which (leaf) grid cells need to be refined, based on certain refinement criteria (see Appendix A.1);

3. refine those cells; and

4. recursively, refine neighboring cells having a lower level of refinement (in order to maintain the gradedness of the grid).

The refinement criteria are described in full detail in Appendix A.1. Roughly, they are based on (1) the differences of phase measured at the vertices and at the center of the cell, (2) the level sets (isochrons) of interest, and (3) the maximum allowed level of refinement. Appendix A also comments on the grid initialization, the computation of level sets, and the gradient computation. In particular, it discusses the issues inherent to the computation of levels sets of the phase function $\Theta$, whose values are not defined on the real line $\mathbb{R}$ but on the circle $\mathbb{S}^{1}$. The criteria and parameters that have been used for the simulations of this paper are given in Appendix B.

4. Global isochrons of the full system. As explained in section 2.3, the subset $\mathcal{S}_{I}$ of the almost phaseless set divides the state space in two regions: the quiescent region and the active bursting region. Trajectories with an initial condition in the quiescent region initially converge toward the quiescent segment of the limit cycle, while trajectories with an initial condition in the active bursting region initially converge toward the active bursting segment. In this section, we separately consider the isochrons in the quiescent region and in the active bursting region, with a particular emphasis on the almost phaseless set.

4.1. Quiescent region. In the quiescent region, the isochrons have a simple geometry (Figure 5), in that the main part of each one is parallel to the $V-n$ plane. Resulting from the slow-fast dynamics of the model, this geometry is reminiscent of the isochrons of the 


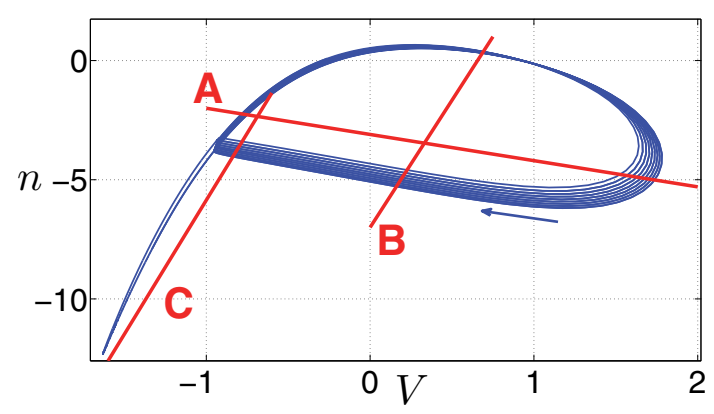

Figure 6. Top view of the cross sections $A, B$, and $C$.

Hodgkin-Huxley model [31], for instance. The other part of each isochron surrounds the bursting segment along the almost phaseless set $\mathcal{S}_{I}$, so that its geometry is determined by the critical manifold $M_{1}^{c}$ (the red curve) and the stable manifold $W^{s}(Q)$ (the magenta curve). Note also that the isochrons do not completely surround the bursting segment, similarly to how the stable manifold $W^{s}\left(\tilde{Q}_{u}\right)$ in a fast subsystem does not completely surround the limit cycle $\tilde{\Gamma}_{s}$.

The stable manifold $W^{s}(Q)$ is the intersection of all the isochrons. This defines a phaseless set, since the trajectories with initial conditions belonging to $W^{s}(Q)$ do not converge to the limit cycle but to the saddle point $Q$.

At the end of the quiescent segment, the geometry of the isochrons becomes more complex, as a sign of the imminent active segment. For instance, the isochrons $\theta=-5 \pi / 6$ and $\theta=$ $-4 \pi / 6$ (the blue and green isochrons in Figure 5) exhibit a complex Z-shaped geometry. They lie at the end of $M_{1}^{c}$ and interfere with $\mathcal{S}_{I I}$, which plays an important role in the active bursting region. In Figure 5, the level of refinement is not sufficient to capture the complete geometry of these isochrons (which is represented with better detail later in Figure 16).

\subsection{Active bursting region.}

4.2.1. Two-dimensional cross sections. Because of the helical geometry of the active bursting segment of the limit cycle, the geometry of the isochrons is more complex there than in the quiescent region. In order to get a global picture of the phase sensitivity in the active bursting region, we first consider the two-dimensional cross sections $\mathrm{A}, \mathrm{B}$, and $\mathrm{C}$ shown in Figure 6. Cross sections $\mathrm{A}$ and $\mathrm{B}$ provide a picture of the isochrons in the entire active bursting region. Cross section $\mathrm{C}$ is considered with low values of the slow variable $h$ and focuses on the beginning of the bursting segment.

Figures 7, 8, and 9 highlight the interplay between the isochrons (panels (a)) and the almost phaseless set (panels (b)) in the three cross sections. In particular, the subset $\mathcal{S}_{I I}$ constrains the geometry of the isochrons in the active bursting region. It also subdivides the active bursting region into smaller subregions, each of which is associated with a spike of the limit cycle, as shown in Figure 7(a). These subregions determine the number of spikes remaining in the burst. We will therefore refer to these regions as spike regions. Figure 10 highlights one isochron that is observed in several spike regions, getting closer to the almost phaseless set as the value of $h$ increases. Note that some isochrons of the quiescent region are 


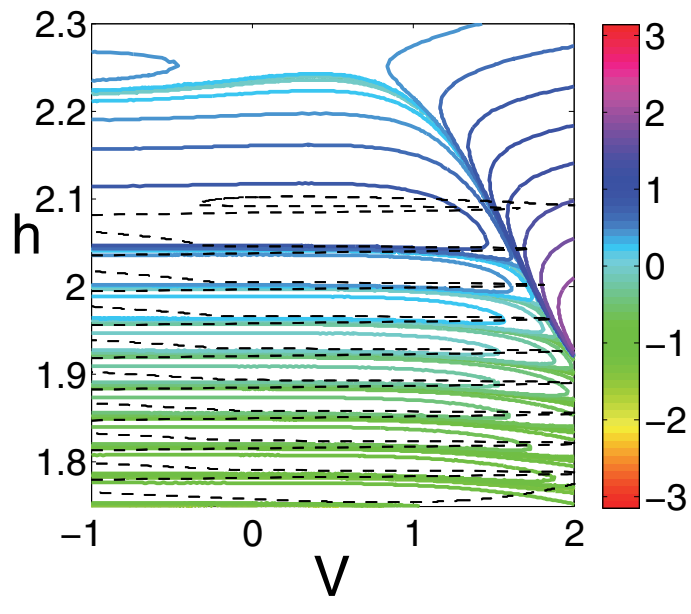

(a)

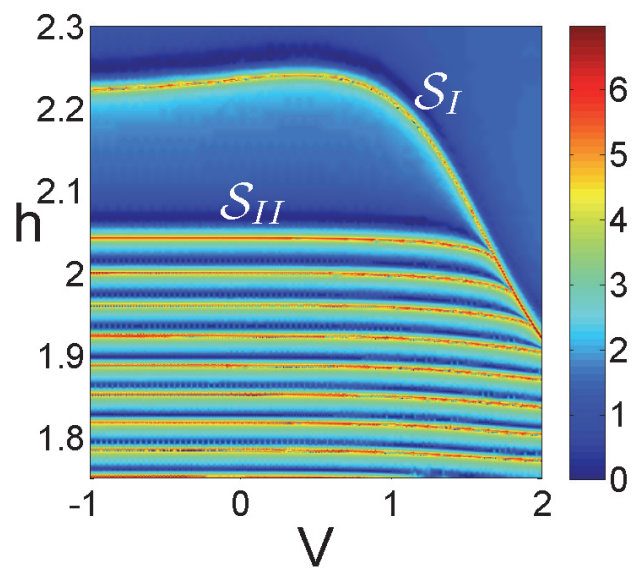

(b)

Figure 7. (a) View of 30 isochrons (equally spaced in phase by $\pi / 15$ ) in cross section A. The black dashed curve is the projection of the limit cycle on cross section A. (b) Gradient of the phase $\nabla \Theta$ (shown as $\max \{0, \ln \|\nabla \Theta\|\})$ in cross section $A$. The set $\mathcal{S}_{I I}$ divides the bursting region into individual spike regions.

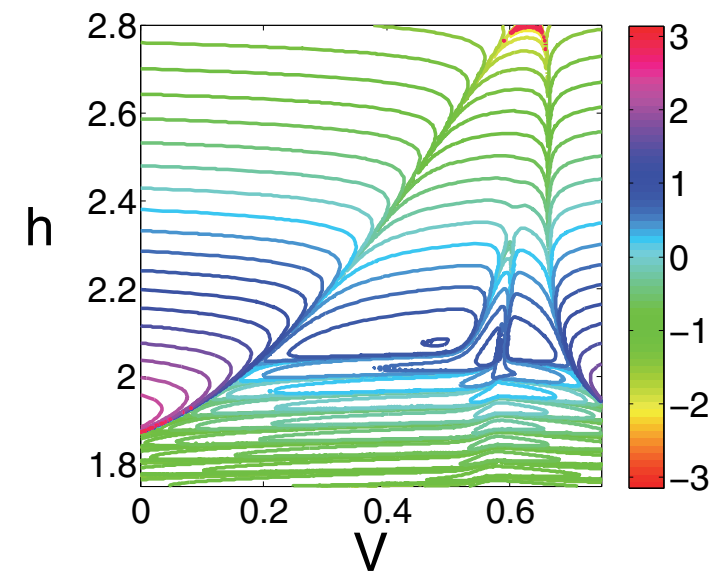

(a)

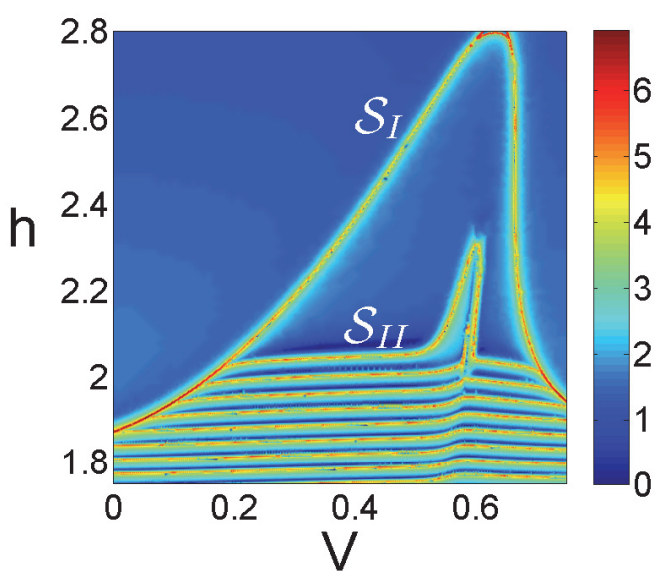

(b)

Figure 8. Same as Figure 7 but for cross section B. The bursting region reaches as high as $h \approx 2.8$, far beyond the limit cycle.

also observed in the last spike region (e.g., the blue curves in Figures 7 and 8). Finally, Figure 11 shows a close-up of the boundary between two spike regions and the quiescent region, where $\mathcal{S}_{I}$ and $\mathcal{S}_{I I}$ merge.

The cross sections provide some information on the global geometry of $\mathcal{S}_{I}$ and therefore on the size of the active bursting region. In particular, we note that $\mathcal{S}_{I}$ reaches as high as $h=2.8$ (Figure 8), far beyond the limit cycle $(h \in[1.7,2.2])$. In contrast, $\mathcal{S}_{I}$ does not extend below the limit cycle, but merges with $\mathcal{S}_{I I}$ at the value $h \approx 1.85$, which corresponds to the 


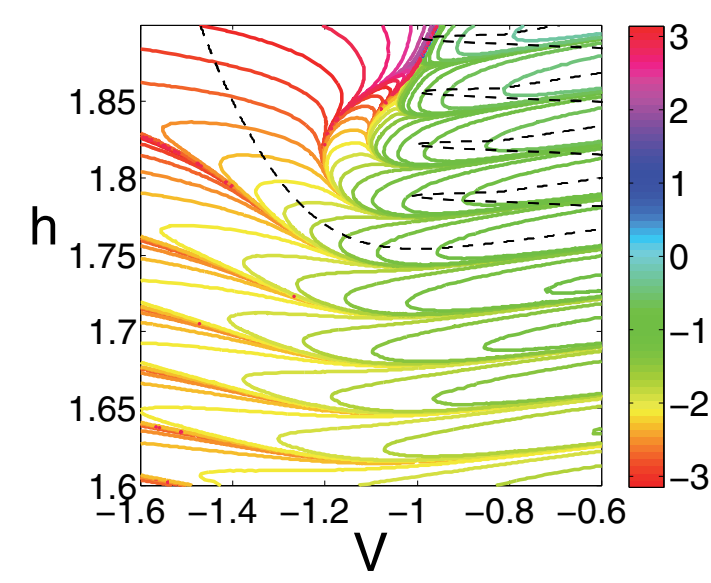

(a)

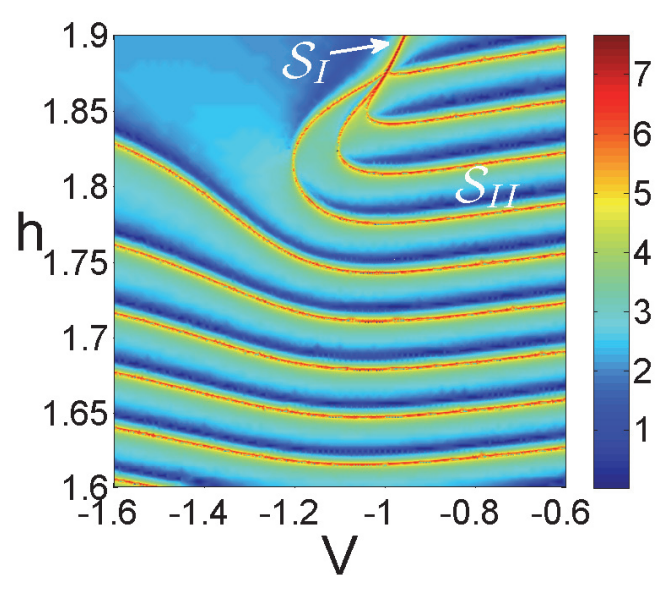

(b)

Figure 9. Same as Figure 7 but for cross section $C$, at the beginning of the bursting segment. The black dashed curve is the projection of the limit cycle on cross section $C$.

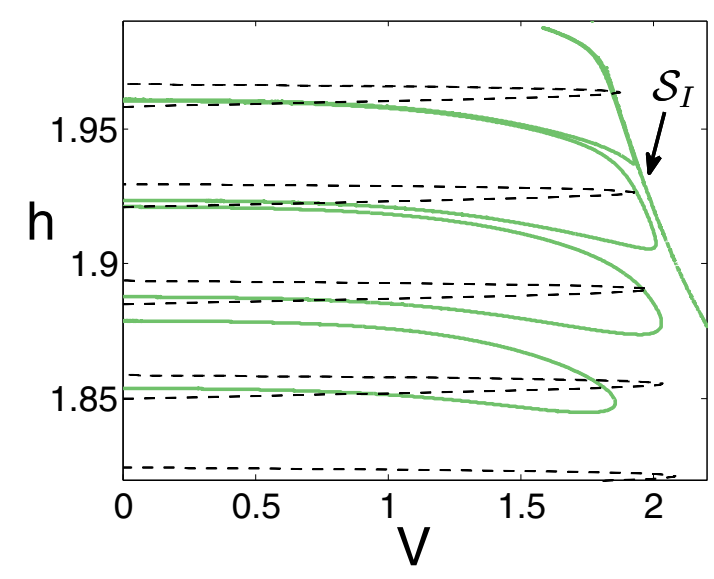

(a)

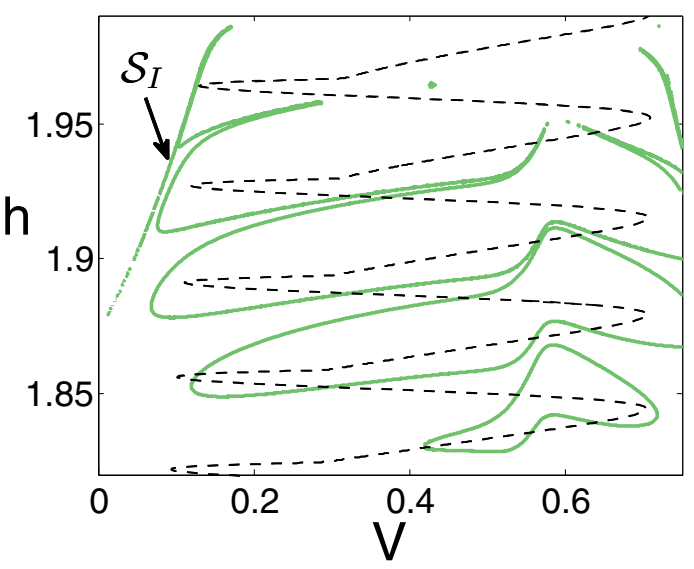

(b)

Figure 10. The isochron $\theta=-0.5$ lies in several spike regions, getting closer to the almost phaseless set $\mathcal{S}_{I}$ as $h$ increases. (a) Cross section A. (b) Cross section B. The black dashed curve is the projection of the limit cycle onto cross sections $A$ and $B$, respectively.

end of the critical manifold $M_{1}^{c}$ (Figure 9). It follows that the active bursting region extends indefinitely below the limit cycle, where $\mathcal{S}_{I I}$ defines spike regions that spread over the whole basin of attraction in the $V$ and $n$ directions. A trajectory starting from this region exhibits several preliminary spikes before reaching the active segment of the limit cycle.

Because of the presence of $\mathcal{S}_{I I}$, the active bursting region is characterized by a high phase sensitivity. This is illustrated by Figure 12, which shows the phase value along the one-dimensional intersection of cross sections A and B. While singular perturbation theory 


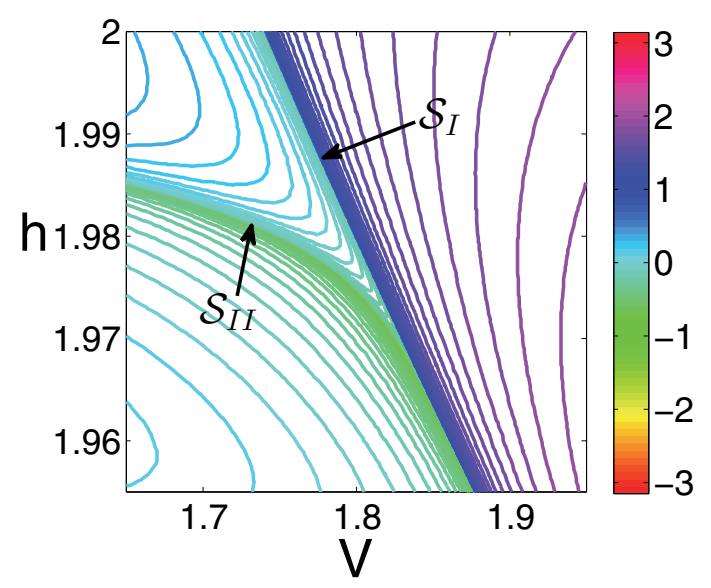

Figure 11. Close-up of a junction of $\mathcal{S}_{I}$ and $\mathcal{S}_{I I}$ in the cross section $A$. The isochrons are spaced in phase by $\pi / 60$.

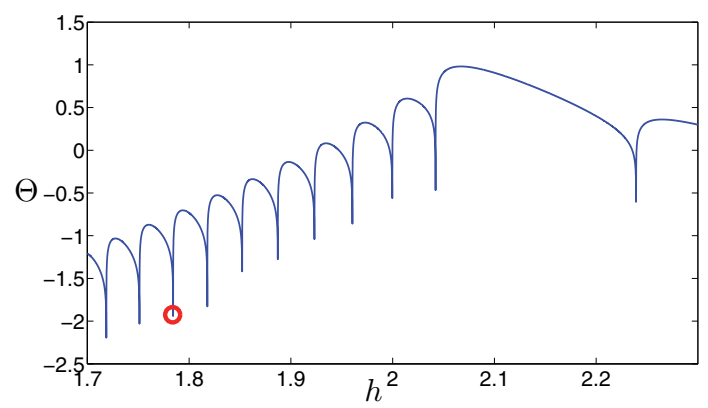

Figure 12. Along the intersection of the cross sections $A$ and $B(V=0.3314, n=-0.3646)$, the evolution of the phase is characterized by sharp negative peaks that account for the crossing of $\mathcal{S}_{I I}$ from one spike region to the next. The red circle corresponds to the initial condition used in Figure 13.

predicts that the phase monotonically increases as $h$ grows, an accurate computation shows that the phase exhibits a succession of sharp negative peaks. These peaks indicate the set $\mathcal{S}_{I I}$ separating two spike regions. In addition, over each spike region, we observe a phase variation of about 0.5 (without considering the sharp peaks), while the average phase increase from one spike region to another is less than 0.2 .

In order to interpret the presence of the set $\mathcal{S}_{I I}$, Figure 13 shows three trajectories with initial conditions in its vicinity (on either side of the peak marked by a red circle in Figure 12). A phase delay appears between the trajectories when they come close to the critical manifold $M_{1}^{c}$, at the end of the bursting segment. At that point, the trajectories split, either entering into the quiescent region (initial condition in the lower spike region) or performing another spike in the last spike region (initial condition in the upper spike region). With an initial condition on the peak, i.e., (almost) on $\mathcal{S}_{I I}$, the black trajectory lingers near the critical manifold, then performs one last spike at a high $h$ value. These observations, which were also reported in [41], show that the set $\mathcal{S}_{I I}$ results from the sensitivity induced by the saddle-type 

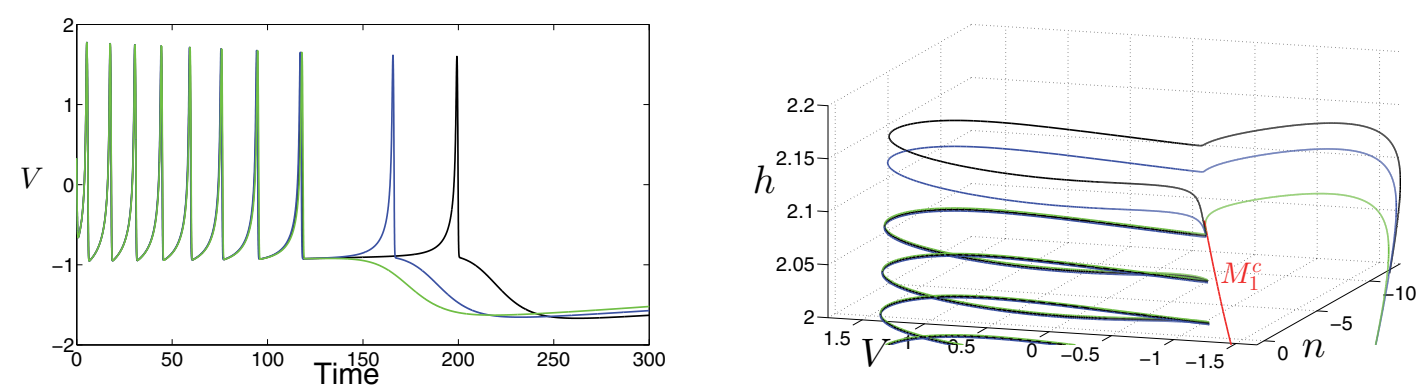

Figure 13. Three trajectories with initial conditions very close to one another but on both sides of $\mathcal{S}_{I I}$ diverge from one another near the critical manifold $M_{1}^{c}$. Trajectories with an initial condition in the upper spike region (blue and black curves) perform one more spike. In particular, the black curve lingers quite a while near the critical manifold. This highlights the high phase sensitivity of the model and explains the effect of $\mathcal{S}_{I I}$. The initial conditions are taken close to the peak marked by a red circle in Figure 12 (i.e., $V=0.3314$, $n=-0.3646, h=1.7843$ (black curve), $h=1.7843-0.001$ (blue curve), $h=1.7843+0.001$ (green curve)).

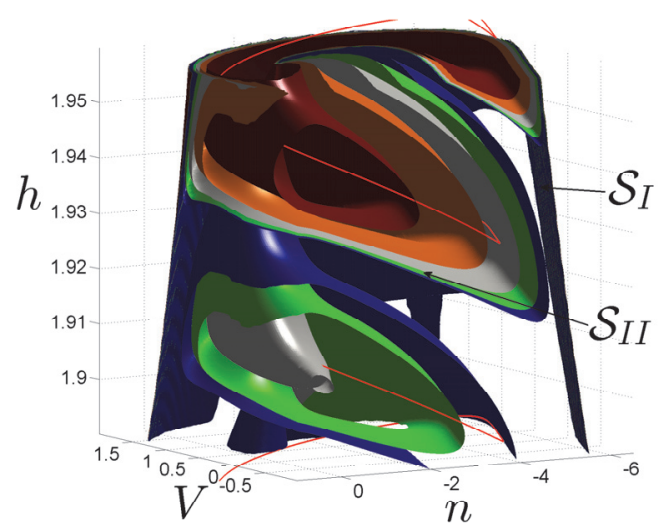

(a)

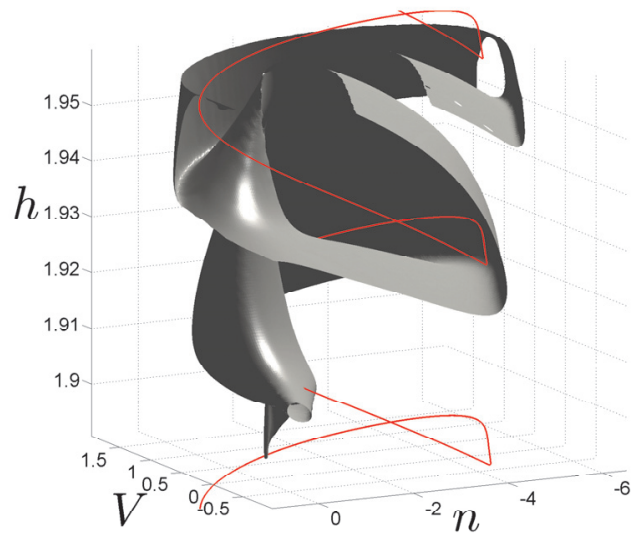

(b)

Figure 14. (a) Isochrons in two spike regions at the middle of the bursting segment. Isochron values: $\theta=-3 \pi / 36$ (blue), $\theta=-2 \pi / 36$ (green), $\theta=-\pi / 36$ (grey), $\theta=0$ (orange), $\theta=\pi / 36$ (red). The red curve is the limit cycle. (b) Isochron $\theta=-\pi / 36$. The part of the isochron lying close to $\mathcal{S}_{I}$ has been suppressed (by removing triangles with edge differences greater than 1.2). See also the associated animation (93115_01.avi [local/web 5.95MB]).

manifold $M_{1}^{c}$.

4.2.2. Three-dimensional views. Now, we compute the two-dimensional isochrons in the active bursting region. Because of their intricate geometry, we do not represent the isochrons in the entire region, but focus on the middle, the beginning, and the end of the bursting segment of the limit cycle.

Middle of the active segment. Figure 14 shows the isochrons in two spike regions at the middle of the active segment. Each isochron intersects the limit cycle only once. However, owing to its complex geometry, part of each isochron remains close to the limit cycle, inside the bursting region delimited by $\mathcal{S}_{I}$. The isochrons intersecting the limit cycle in the lower spike 


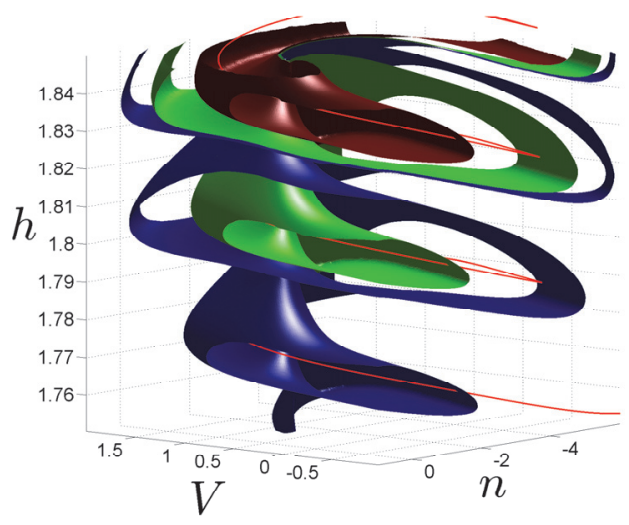

Figure 15. The isochrons at the beginning of the bursting segment (here in three spike regions) are similar to the isochrons at the middle of the bursting segment (shown in Figure 14). However, they are less concentrated, so that the phase sensitivity is lower. Isochron values: $\theta=-5 \pi / 18$ (blue), $\theta=-4 \pi / 18$ (green), $\theta=-3 \pi / 18$ (red). The red curve is the limit cycle. See also the associated animation (93115_02.avi [local/web 5.97MB]).

region also appear in the upper spike region, where they are closer to the almost phaseless set.

The closer an isochron lies to the center of a spike region, the higher the phase value associated with it. This is explained by the fact that trajectories starting from the center of a spike region, i.e., near the unstable fixed point of the fast subsystem and toward the middle in the $h$ direction, exhibit a shorter and faster first spike, and are therefore characterized by a slight phase advance with respect to trajectories on the limit cycle. This observation confirms the result in [41] showing that a perturbation pushing the trajectory inside the bursting segment will induce a phase advance (and vice versa). This also explains the presence of maxima in Figure 12.

Figure 14(b) depicts a single isochron and shows how it evolves around the limit cycle (see also the corresponding animation (93115_01.avi [local/web 5.95MB])). After the intersection with the limit cycle, the isochron surrounds an entire spike of the limit cycle. Above this spike, the intersection of $\mathcal{S}_{I I}$ with the isochron creates an "opening" in the isochron, so that the limit cycle can escape the isochron (without intersecting a second time) and enter into the next spike region.

Inside the bursting segment, the set $\mathcal{S}_{I I}$ is characterized by a helicoidal geometry. This is intimately related to the trajectories (and in particular the limit cycle), forcing them to spiral from one spike region to the next.

Beginning of the active segment. The isochrons lying at the beginning of the active bursting segment (Figure 15; see also the corresponding animation (93115_02.avi [local/web 5.97MB])) are similar to those in the middle of the active segment (Figure 14). A noticeable difference, though, is that part $\mathcal{S}_{I}$ of the almost phaseless set is far from the limit cycle (not seen in Figure 14), so that the isochrons are less concentrated. It follows that the phase sensitivity is higher in the middle of the bursting segment than at the beginning. This is in accordance with the results of [41], showing that the phase sensitivity increases with proximity to the end of the active bursting segment. 


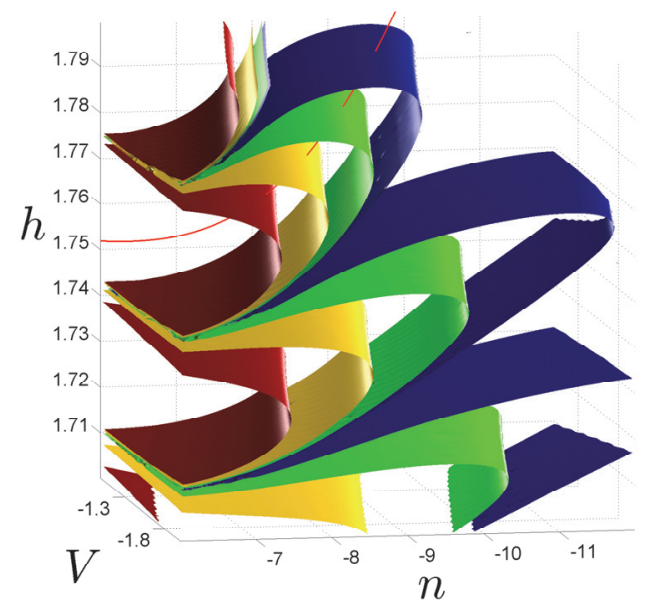

Figure 16. Isochrons at the end of the quiescent segment (corresponding to the bottom right of Figure 5). The limit cycle enters into the bursting region between two slices of $\mathcal{S}_{I I}$. Isochron values: $\theta=-12 \pi / 18$ (blue), $\theta=-11 \pi / 18$ (green), $\theta=-10 \pi / 18$ (yellow), $\theta=-9 \pi / 18$ (red). The red curve is the limit cycle.

Figure 16 shows the isochrons at the end of the quiescent segment, near the active bursting region. The isochrons concentrate along $\mathcal{S}_{I I}$, which corresponds in the figure to three parallel slices. The limit cycle appears between two of these slices, thereby entering into the active bursting region. Note that these isochrons are the Z-shaped isochrons observed in the bottom right of Figure 5.

End of the active segment. At the end of the active segment, the part $\mathcal{S}_{I}$ of the almost phaseless set delimits the bursting region in which the limit cycle seems to be trapped (a situation that is not observed at the beginning of the active segment). Since the limit cycle cannot cross the almost phaseless set, it is of interest to investigate how it comes out of the bursting region and enters into the quiescent region.

The isochrons at the end of the active segment are represented in Figure 17. They are similar to the isochrons in the middle of the active segment (Figure 14), but it can be seen in Figure $17(\mathrm{~b})$ that $\mathcal{S}_{I}$ and $\mathcal{S}_{I I}$ merge together and wrap up, creating a small vertical cylinder (see also the corresponding animation (93115_03.avi [local/web 2.69MB])). The limit cycle turns around this cylinder during the last spike and then leaves the bursting region.

4.2.3. Onset and termination of the burst, spike addition and deletion. The almost phaseless sets can be used to investigate (and control) the effect of particular perturbations on the initiation and termination of the burst and on the number of spikes of the burst. Indeed, the subset $\mathcal{S}_{I}$ of the almost phaseless set is a boundary between the bursting region and the quiescent region. If, under the effect of a perturbation, a trajectory crosses $\mathcal{S}_{I}$, an early burst initiation or termination will be observed. For high values of $h, \mathcal{S}_{I}$ is close to the limit cycle, so that a small perturbation is sufficient to terminate the bursting (see also section 5). Similarly, the part $\mathcal{S}_{I I}$ of the almost phaseless set is a boundary between the spike regions. If the trajectory crosses $\mathcal{S}_{I I}$, one (or more) spike(s) will be added to or deleted from the burst. In addition, a perturbation pushing the trajectory below the limit cycle (i.e., in the 


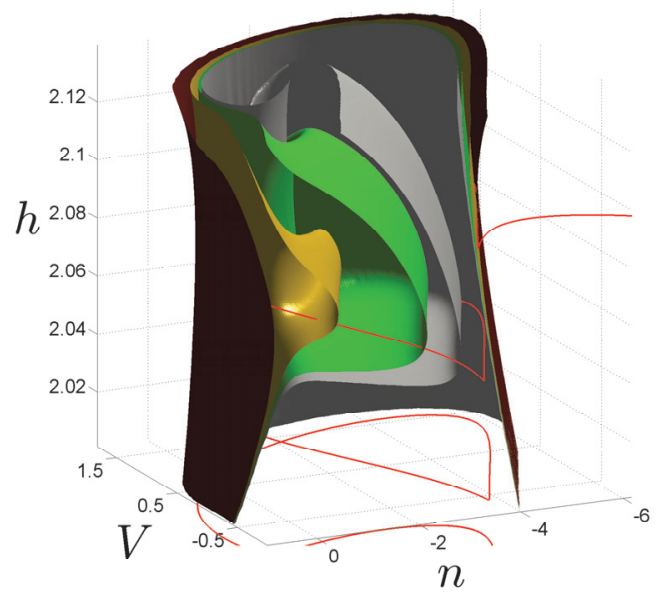

(a)

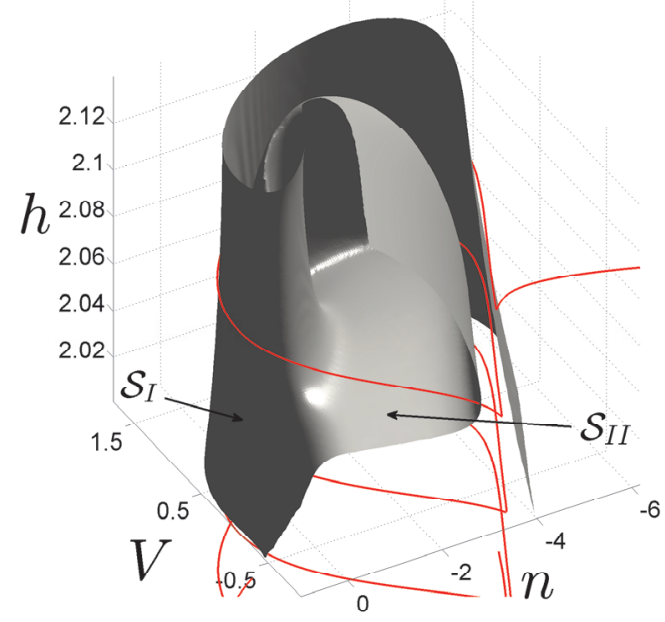

(b)

Figure 17. Isochrons in the last spike region, at the end of the bursting segment. (a) The limit cycle intersects the grey, green, and yellow isochrons in the bursting region and intersects the red isochron in the quiescent region. Isochron values: $\theta=10 \pi / 36$ (grey), $\theta=11 \pi / 36$ (green), $\theta=12 \pi / 36$ (yellow), $\theta=13 \pi / 36$ (red). The red curve is the limit cycle. (b) Isochron $\theta=10 \pi / 36$. The sets $\mathcal{S}_{I}$ and $\mathcal{S}_{I I}$ merge together, creating a small cylinder that the limit cycle encircles when leaving the bursting region during the last spike. Regions of the isochron lying close to $\mathcal{S}_{I}$ and $\mathcal{S}_{I I}$ are marked with arrows. See also the associated animation (93115_03.avi [local/web 2.69MB]).

region $h<1.8$; see Figure 9) will induce a longer burst, characterized by additional spikes. In this context, all the situations considered in [41] for obtaining the different phenomena (burst initiation and termination, spike addition and deletion) correspond to the crossing of one part - $\mathcal{S}_{I}$ or $\mathcal{S}_{I I}$ - of the almost phaseless set. In the next section, we investigate in more detail the phase sensitivity and its relationship to the almost phaseless set, focusing on the subset of the state space defined by certain perturbations of limit cycle states.

5. Phase response. Exploiting the flexibility of our method, we now investigate the phase response of the model (see the definitions of PRF and PRE in section 2.2). For this purpose, we focus the computation on the two-dimensional surface of states reachable from the limit cycle via impulse inputs of size $|\alpha|<0.75$ in the voltage state $V$. More precisely, the grid is defined on a domain $[0,1] \times[-0.75,0.75]$ of values $(\tilde{\theta}, \alpha)$ that corresponds to a two-dimensional strip of (perturbed) states $\mathbf{x}^{\gamma}+\alpha \mathbf{e}$ (with $\left.\mathbf{e}=(1,0,0)\right)$. The use of a reparametrization $\tilde{\theta}=\tilde{\theta}(\theta)$ of the limit cycle enables us to better refine the active bursting region (see Figure 18). This reparametrization is based on a cumulative distance along the limit cycle in the state space, in which the weight on each of the three state components is inversely proportional to their range (along the limit cycle). The quadtree grid is refined based on the PRF $Z$ instead of $\Theta$, but also provides a satisfactory picture of $\Theta$, without any additional computation.

Figure 18 shows the phase function and the PRF on the described two-dimensional surface in state space and highlights the almost phaseless set as the boundary between areas of different 


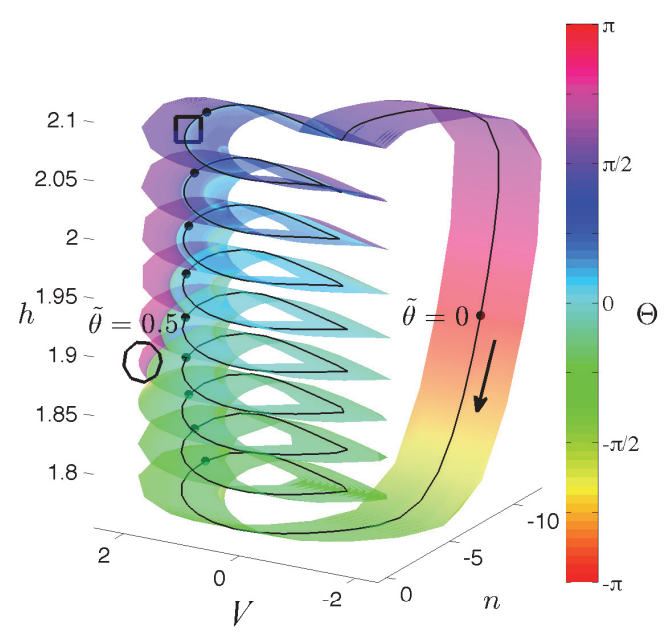

(a)

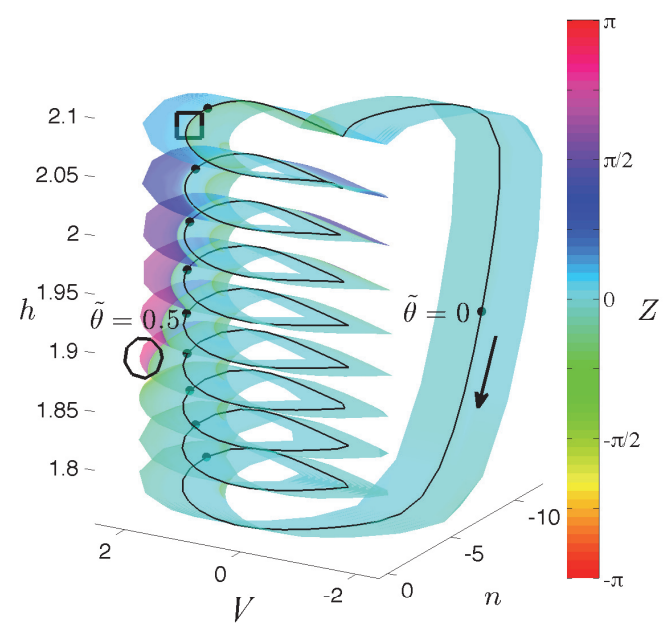

(b)

Figure 18. Phase function $\Theta$ (a) and phase response function $Z$ (b) on the surface of states potentially reachable from the limit cycle by an impulsive perturbation of size $\alpha \in[-0.75,0.75]$. The almost phaseless set $\mathcal{S}_{\mathcal{I}}$ is indicated by jumps in color value. For example, a perturbation that pushes the trajectory across $\mathcal{S}_{\mathcal{I}}$ into the lower left pink area (black circle) effectively terminates the burst. The most efficient perturbation-see below-is triggered in the black square. The limit cycle is shown in black, and the ten black dots indicate steps of 0.1 in the variable $\tilde{\theta}$.

color. For instance, $\mathcal{S}_{I}$ appears as the boundary between the green and pink areas, near the left-hand edge (black circle). One observes that it gets closer to the bursting segment of the limit cycle as $h$ increases from 1.9 to 2.1 , where it finally overtakes the limit cycle and merges with $\mathcal{S}_{I I}$ (see also the same observation in section 4.2.2). The practical significance of this observation is that, as already shown in [41], a positive voltage perturbation of intensity less than $\alpha_{\max }=0.75$, in the fourth spike (black circle in Figure $18, h \approx 1.9$ ), effectively terminates the burst, pushing the trajectory across $\mathcal{S}_{I}$ and into the pink area (see also Figure 22 (a) below). In addition, even smaller perturbations terminate the burst, albeit in later spikes.

Figure 19 depicts the PRF $Z$ (in log scale) in the $(\tilde{\theta}, \alpha)$ plane. The largest observed phase responses are indicated by the purple and pink regions, which all correspond to perturbations across $\mathcal{S}_{1}$ (marked by the black arrows), and thus into the quiescent region. Note that the four light blue and green regions near $\tilde{\theta}=0.9$ (where the limit cycle is already in the quiescent region) correspond, respectively, to phase advances induced by outward perturbations that push the trajectories far from the bursting segment, and to phase delays induced by inward perturbations that push the trajectories across $\mathcal{S}_{I}$ and back into the bursting region. The other, lighter, thinner blue and green regions indicate weaker phase shifts induced by the crossing of $\mathcal{S}_{I I}$, each adding or deleting a single spike.

Figure 19 shows the large phase difference for the perturbation mentioned above, induced by a (quite small) impulse $\alpha \approx 0.5$ near $\tilde{\theta}=0.4$ (see also Figure 22(a)). In addition, a significant phase difference of size $|Z| \approx \pi / 2$ can be obtained for very small voltage impulses 


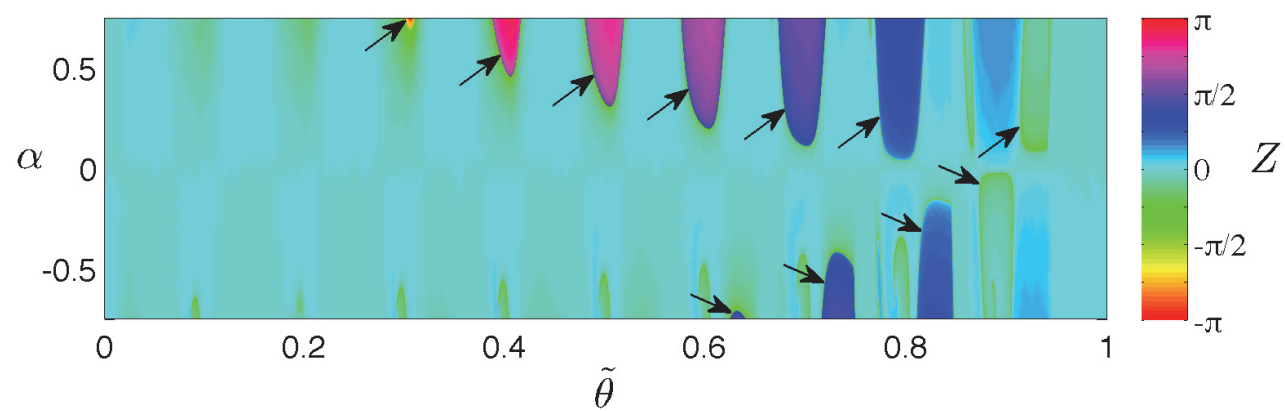

Figure 19. The phase response function $Z$ for impulse voltage differences of size $\alpha \in[-0.75,0.75]$. Phase shifts of almost $\pi$ can be obtained with perturbations of size $\alpha \approx 0.5$. The black arrows point to the boundaries between two regions of different colors that correspond to the crossing of $\mathcal{S}_{I}$.

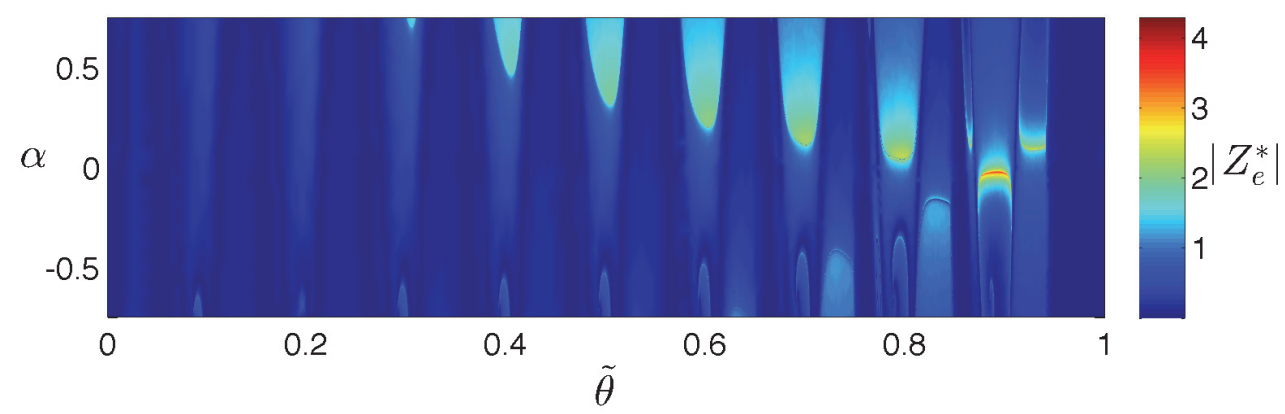

Figure 20. The PRE (or rather its logarithmically scaled magnitude $\left|Z_{e}^{*}\right|=\ln \left(\left|Z_{e}\right|+1\right)$ ) has a global maximum of $\approx 4.29\left(Z_{e}=71.91\right)$ for $\tilde{\theta} \approx 0.89$ (or $\theta \approx 1.10$ ) and $\alpha \approx-0.0215$ (in red), which corresponds to the most efficient perturbation.

$\alpha<0.1$ (near $\tilde{\theta}=0.8$ ), owing to the proximity of $\mathcal{S}_{I}$ to the limit cycle. These weaker phase shifts are captured more clearly in terms of the PRE $Z_{e}$ - the "efficiency" of the perturbation.

Figure 20 depicts the PRE $Z_{e}$ (in log scale) in the $(\tilde{\theta}, \alpha)$ plane. A global maximum of $Z_{e}=71.91$ is obtained near $\tilde{\theta}=0.89(\theta=1.10)$ and $\alpha \approx-0.0215$. (Note that $Z<0$ but is divided by $\alpha<0$, to yield $Z_{e}>0$.) This global maximum is related to the most efficient perturbation. It corresponds to a perturbation towards the center of the bursting region applied during the final spike (black square in Figure 18), where the limit cycle is very close to-but outside - the subset $\mathcal{S}_{I}$. Under the effect of the perturbation, the trajectory crosses back over $\mathcal{S}_{I}$ and repeats the last spike (Figure 22(b)). Also visible here (in yellow) but not as prominent are the aforementioned extrema of $Z$ that require larger values of $\alpha$.

Figure 21 shows $Z_{e}$ (scaled logarithmically), versus $\tilde{\theta}$ and $\theta$, along one-dimensional slices at $\alpha=0,-0.01,-0.02$, and -0.03 . These curves are coarse but are extracted virtually for free through quadratic triangle-based interpolation. They exhibit a peak that jumps from $Z_{e} \approx 9.06$ to $Z_{e} \approx 48.33$ as $\alpha$ goes from -0.01 to -0.02 . The $\alpha=0$ curve - computed as a directional derivative of $\Theta$, according to (2.7) - serves as a coarse approximation of the iPRC 


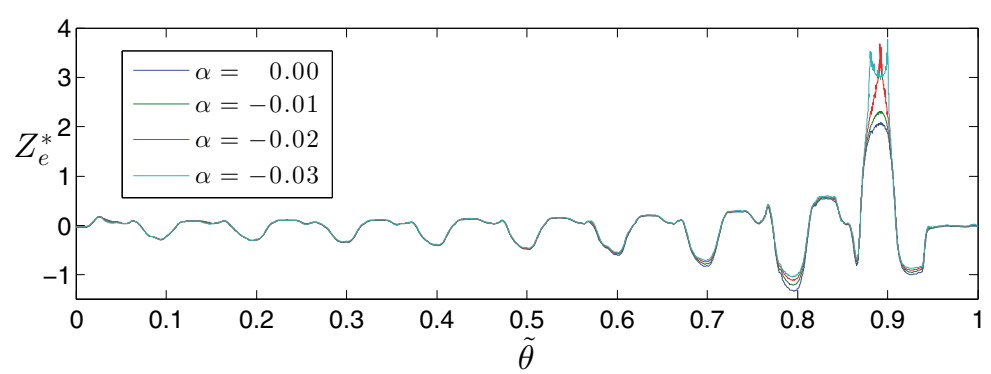

(a)

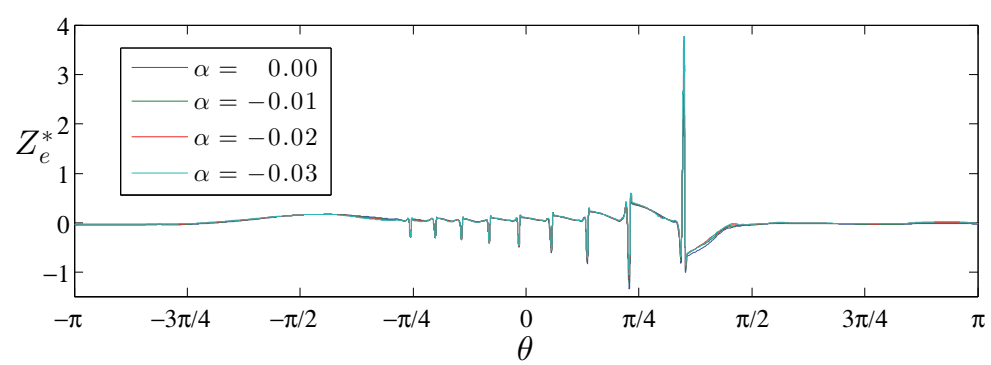

(b)

Figure 21. $P R E$ (or rather the logarithmically scaled version $Z_{e}^{*}=\operatorname{sgn}\left(Z_{e}\right) \ln \left(\left|Z_{e}\right|+1\right)$ ) versus (a) $\tilde{\theta}$ and (b) $\theta$, along one-dimensional slices. The global maximum of $Z_{e}^{*} \approx 4.29\left(Z_{e}=71.91\right)$ for $\tilde{\theta} \approx 0.89$ (or $\theta \approx 1.10$ ) and $\alpha \approx-0.0215$ (in Figure 20) is highlighted by the jump of the main peak from $Z_{e}^{*}=2.31\left(Z_{e}=9.06\right)$ to $Z_{e}^{*}=3.69\left(Z_{e}=48.33\right)$ as $\alpha$ goes from -0.01 to -0.02 . The curve $\alpha=0$ is the iPRC, also computed in $[33,41]$.

and compares well with the iPRC computed in [41, Figure 4, p. 671] and [33, Figure 4, p. 524]. It is in fact itself characterized by a global maximum of 7.01 near $\tilde{\theta}=0.89$ (or $\theta=1.10$ ), which is fairly high, but still significantly lower than that of $\alpha=-0.02$, which results from the crossing of $\mathcal{S}_{I}$. As hinted before, the proximity of this highly phase-sensitive feature to $\alpha=0$-i.e., to the limit cycle - is one of the more remarkable aspects of bursting neuron models in general. (Note also that similar phase response curves were computed in [10] for a square-wave bursting model in response to particular continuous stimuli.)

In conclusion, the computation of the PRF and the PRE (through the method proposed in [31] and on a quadtree-based adaptive grid) enables us to design the best perturbations for large phase shifts (Figure 22(a)) and the most efficient perturbations (Figure 22(b)). And in every case, the optimal strategy corresponds to the crossing of $\mathcal{S}_{I}$.

6. Phase sensitivity of an elliptic bursting model. In this section, we briefly investigate the phase sensitivity of an elliptic bursting model (or type III bursting model). In particular, we focus on the model proposed in [37], i.e., the Morris-Lecar dynamics with an additional slow variable. The dynamics are given by

$$
\begin{aligned}
C \dot{V} & =-g_{C a} m_{\infty}(V)\left(V-V_{C a}\right)-g_{K} n\left(V-V_{K}\right)-g_{L}\left(V-V_{L}\right)-g_{K C a} z(h)\left(V-V_{K}\right)+I, \\
\dot{n} & =\phi\left(n_{\infty}(V)-n\right) / \tau(V), \\
\dot{h} & =\epsilon\left(-\mu g_{C a} m_{\infty}(V)\left(V-V_{C a}\right)-h\right),
\end{aligned}
$$




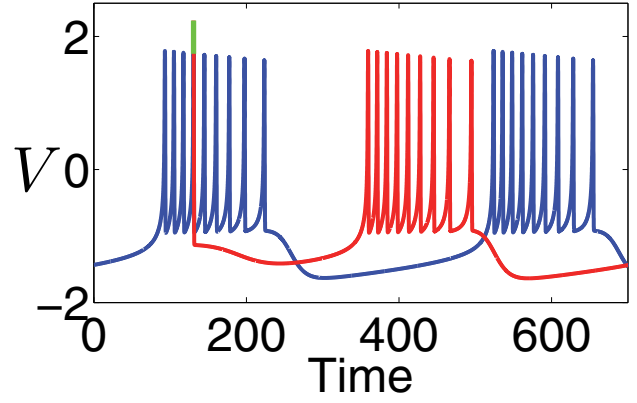

(a)

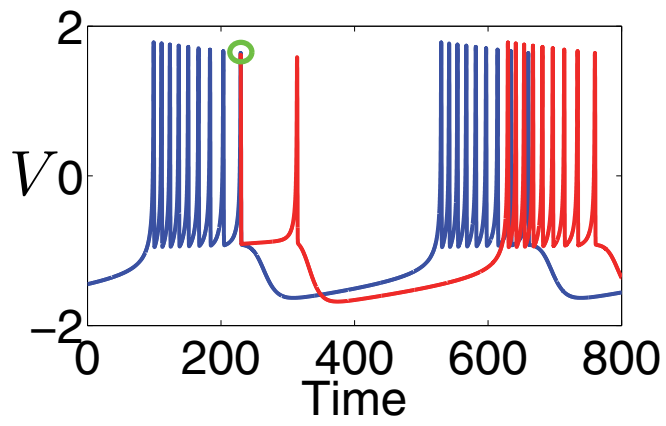

(b)

Figure 22. Comparison of perturbed (red) and unperturbed (blue) trajectories. (a) Perturbation for large phase shift (high value of $Z$ ). A (quite small) perturbation in the middle of the bursting segment terminates the burst, inducing a large phase difference. The perturbation (green) has an intensity $\alpha=0.5$ and occurs on the limit cycle at $(V, n, h)=(1.7392,-4.042,1.888)$ (black circle in Figure 18). (b) Most efficient perturbation (global maximum of $Z_{e}$ ). A very small perturbation during the last spike of the burst adds an additional spike, inducing a significant phase lag. The perturbation has an intensity $\alpha=-0.0215$ and occurs on the limit cycle at $(V, n, h)=(1.5563,-2.2827,2.0848)$ (green circle in the figure, black square in Figure 18).

with

$$
\begin{aligned}
m_{\infty}(V) & =0.5\left(1+\tanh \frac{V+1.2}{18}\right), \\
n_{\infty}(V) & =0.5\left(1+\tanh \frac{V-2}{30}\right), \\
z(h) & =\frac{h}{1+h}, \\
\tau(V) & =1 / \cosh \frac{V-2}{60} .
\end{aligned}
$$

The variable $V$ is the membrane voltage; $n$ and $h$ are the (potassium and calcium) ion currents. Apart from a slight modification, the parameters are taken from [2, 37]; i.e., $C=10 \mu \mathrm{F} / \mathrm{cm}^{2}$, $g_{C a}=4 \mathrm{mS} / \mathrm{cm}^{2}, g_{K}=8 \mathrm{mS} / \mathrm{cm}^{2}, g_{L}=2 \mathrm{mS} / \mathrm{cm}^{2}, V_{K}=-84 \mathrm{mV}, V_{L}=-60 \mathrm{mV} ; V_{C a}=$ $120 \mathrm{mV}, I=120 \mu \mathrm{A} / \mathrm{cm}^{2}, g_{K C a}=0.75 \mathrm{mS} / \mathrm{cm}^{2}, \phi=0.04, \epsilon=0.002$, and $\mu=0.3$. With these parameters, $h$ is a slow variable, and the dynamics of the fast subsystems are the Morris-Lecar dynamics (6.1)-(6.2). The full dynamics admit a stable limit cycle of period $T_{0} \approx 1697.6$, characterized by a quiescent segment inside the bursting segment (see Figure $23)$. The system has also a saddle point $Q$ (at the state $(V, h, n) \approx(-24.073,0.150,12.621)$ ) that lies close to (but below) the limit cycle (at the end of the quiescent segment). It is also noticeable that the stable manifold $M^{s}(Q)$ of the saddle point is very close to the critical manifold $M^{c}$-i.e., the line of stable fixed points $\tilde{Q}_{s}$ of the fast subsystems - which is tracked by the quiescent segment.

In the case of elliptic bursting, each fast subsystem admits an unstable limit cycle $\tilde{\Gamma}_{u}$ (as shown in Figure 24(a)), which is involved in the bifurcations underlying the bursting mechanism (see, e.g., $[25,28]$ ). The termination of the active bursting is induced by a fold 


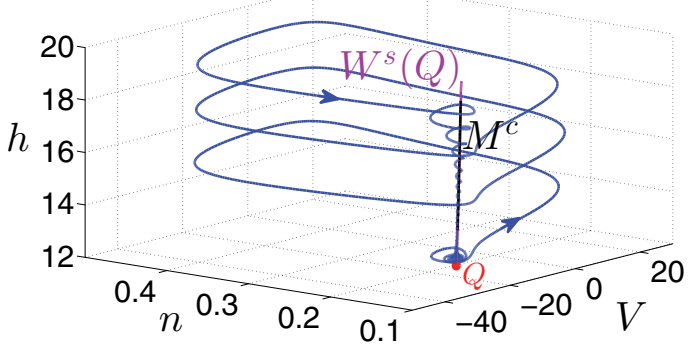

(a)

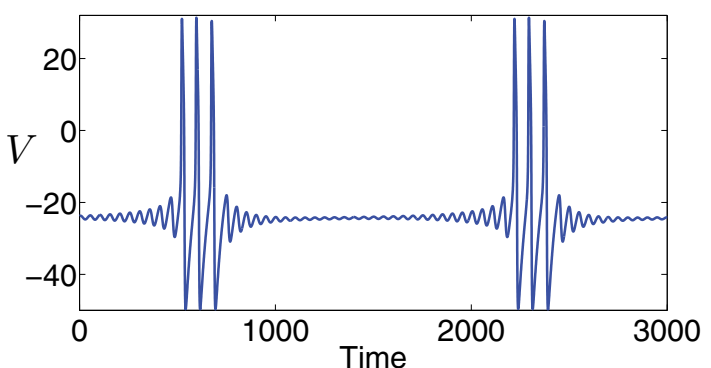

(b)

Figure 23. The dynamics (6.1)-(6.3) exhibit a periodic bursting behavior (elliptic bursting). (a) Limit cycle in the state space, with the stable manifold $W^{s}(Q)$ of the saddle point $Q$ and the critical manifold $M_{c}$. (b) Periodic evolution of the membrane voltage $V$.

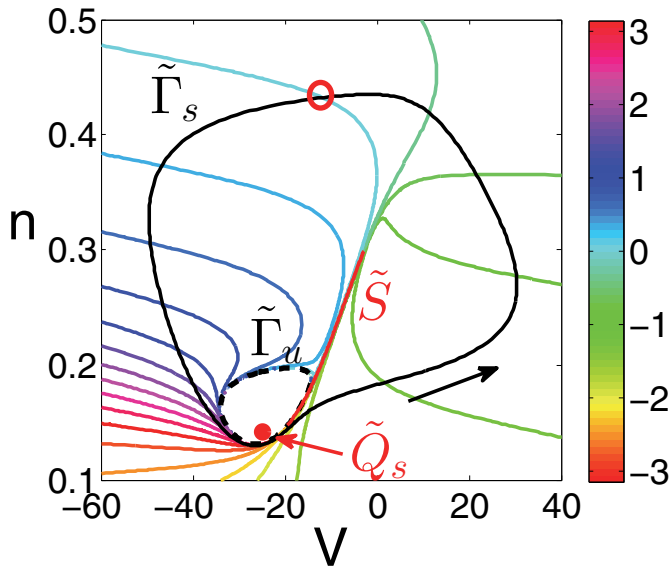

(a)

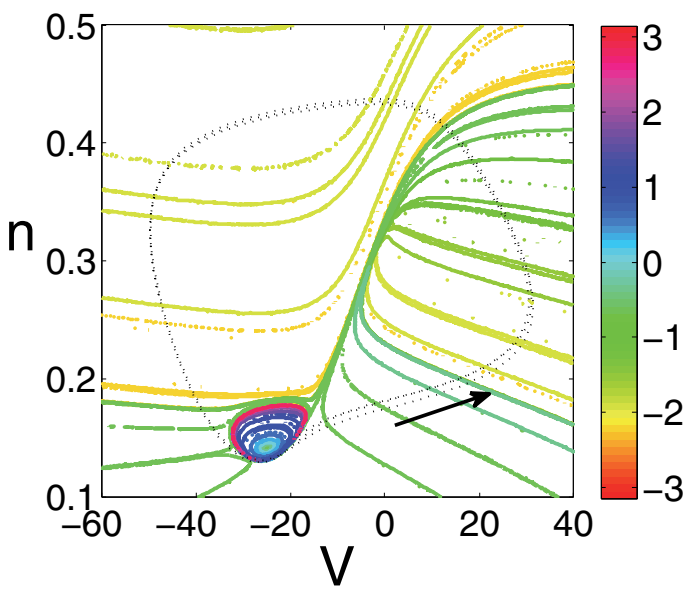

(b)

Figure 24. The isochrons of the fast subsystem for $h=17$ (a) are compared with the corresponding cross section of the isochrons of the original system (b). In the fast subsystem (Morris-Lecar dynamics), the isochrons (spaced in phase by $\pi / 10$ ) concentrate near the unstable limit cycle $\tilde{\Gamma}_{u}$ and near the slow manifold $\tilde{S}$. The red circle represents the point of the limit cycle associated with a phase $\theta=0$. (A similar picture of the isochrons of the Morris-Lecar model can be found in [20, Figure 7].) In the full system, the geometry of the isochrons (spaced in phase by $\pi / 10$ ) is much more complex. The disconnected points observed in the figure are due to the fractal geometry of the isochrons, discussed below. The dashed black curve is the bursting segment of the limit cycle projected onto the cross section.

bifurcation of $\tilde{\Gamma}_{u}$ and the stable limit cycle $\tilde{\Gamma}_{s}(h \approx 18$ for $(6.1)-(6.3))$. In addition, the termination of the quiescent state is induced by a subcritical Hopf bifurcation of $\tilde{\Gamma}_{u}$ and $\tilde{Q}_{s}$ $(h \approx 14)$.

As done in section 2.3 for the HR model (see Figure 2), the isochrons of the fast subsystem (6.1)-(6.2) with $h=17$ are compared with the $h=17$ cross section of the isochrons of the full system (Figure 24). In the fast subsystem (Figure 24(a)), the unstable limit cycle $\tilde{\Gamma}_{u}$ is the 


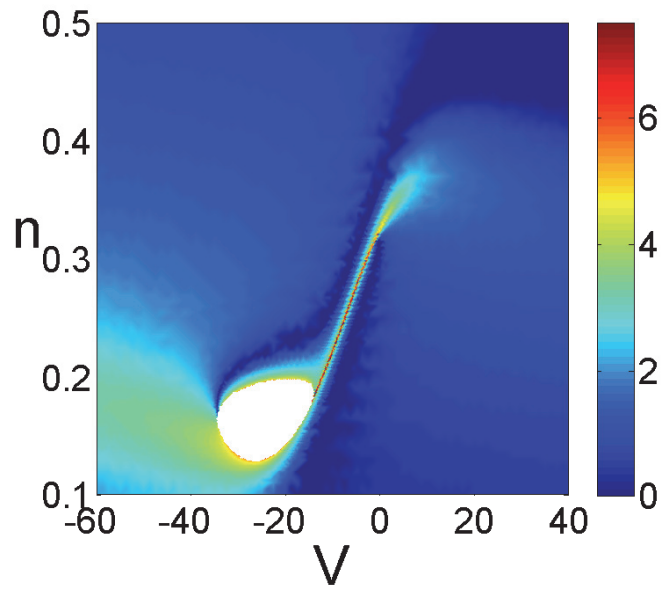

(a)

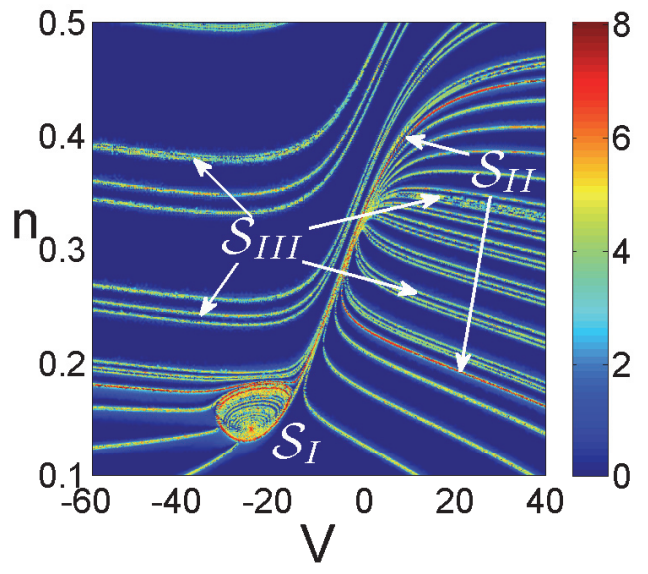

(b)

Figure 25. The gradient of the phase function $\nabla \Theta$ (shown as $\max \{0, \ln \|\nabla \Theta\|\})$ is computed in the fast subsystem for $h=17$ (a) and in the corresponding slice for the full system (b). In the fast subsystem, the almost phaseless sets $\mathcal{S}_{I I}$ and $\mathcal{S}_{I I I}$ are not observed. For the full system, the almost phaseless set $\mathcal{S}$ lies in a large part of the state space, and the quiescent region is characterized by a high gradient value.

boundary between the basins of attraction of $\tilde{Q}_{s}$ and $\tilde{\Gamma}_{s}$. (Note that the phase is not defined inside $\tilde{\Gamma}_{u}$.) The isochrons therefore concentrate near $\tilde{\Gamma}_{u}$. In addition, the (Morris-Lecar) dynamics of the subsystem are themselves slow-fast, so that a concentration of isochrons is also observed along the slow manifold $\tilde{S}$ which separates the trajectories converging to different regions of the limit cycle (the same is observed with the Hodgkin-Huxley model [34]). The presence of the unstable limit cycle and the slow manifold is a one of the main differences between the fast subsystems of this elliptic bursting model and those of the HR model. In the full system (Figure 24(b)), the isochrons have a geometry reminiscent of the isochrons of the fast subsystems but are clearly much more complex. Even though they concentrate in the regions corresponding to $\tilde{\Gamma}_{u}$ and $\tilde{S}$ in the fast subsystem, they also concentrate in other regions. Even more than in the case of the HR model, the complete geometry of the isochrons of the full system is not predicted by singular perturbation theory. This illustrates the importance of performing the phase analysis for the full system.

The gradient of the phase, shown in Figure 25, highlights the regions characterized by a high concentration of isochrons, therefore providing information on the almost phaseless set in the full system. The subset $\mathcal{S}_{I}$ of the almost phaseless set is the boundary between the quiescent region and the bursting region. It is predicted by the fast subsystems, where it corresponds to $\tilde{\Gamma}_{u}$. The set $\mathcal{S}_{I I}$ is characterized by two disconnected parts in the cross section $h=17$. Even though one part is in the prolongation of the slow manifold $\tilde{S}$ of the fast subsystem, it is not predicted by the fast subsystem. As in the HR model, numerical simulations show that $\mathcal{S}_{I I}$ corresponds to the boundary between two spike regions. Also, trajectories with initial conditions close to $\mathcal{S}_{I I}$ but in two different spike regions split at the end of the bursting segment. This phenomenon is very similar to the one depicted in Figure 


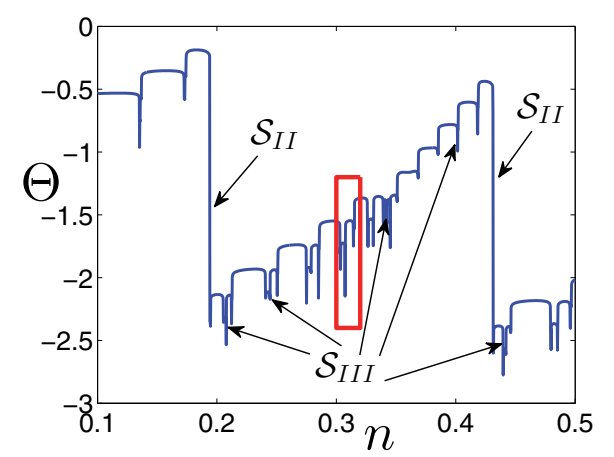

(a)

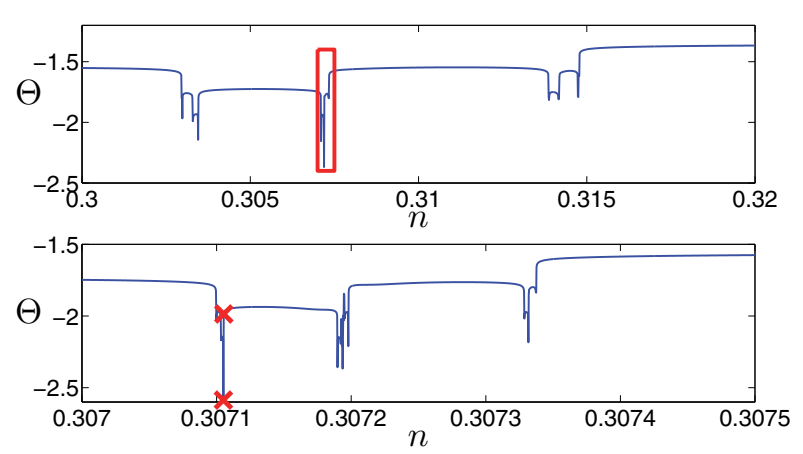

(b)

Figure 26. (a) The phase versus $n$ in the one-dimensional slice $(V, h)=(20,17)$ shows the high phase sensitivity of the elliptic bursting model. In particular, one finds very irregular and sharp peaks and valleysthe regions associated with $\mathcal{S}_{I I I}$-separated by longer regions of almost constant phase. This contrasts with Figure 12, obtained with the HR model. (b) A close-up of the phase profile (in the red rectangles) highlights the fractal geometry of $\mathcal{S}_{I I I}$. (The red crosses correspond to initial conditions used in Figure 27.)

13 for the HR model and is not shown here.

Figure 25(b) shows that the elliptic bursting model exhibits an important difference from the HR model. The (almost) phaseless set admits a third part $\mathcal{S}_{I I I}$ that covers a large portion of the state space, an observation which reveals the high phase sensitivity of elliptic bursting. In contrast with the HR model, this can be explained by the fact that the quiescent region is very sensitive, as illustrated by the high gradient value in this region. In particular, the quiescent region contains the phaseless set $W^{s}(Q)$, and since $M^{c}$ is close to $W^{s}(Q)$, the quiescent segment lies in a small cylinder around $W^{s}(Q)$, where the concentration of isochrons is arbitrarily high. Moreover, the end of the quiescent segment is particularly sensitive, since it is confined in a small region close to the unstable fixed point $Q$ (see Figure 23(a)).

For the elliptic bursting model, the phase value versus $n$ in the one-dimensional slice $(V, h)=(20,17)$ (Figure 26(a)) is much different from that of the HR model (Figure 12). It is characterized by alternating intervals of (almost) constant phase, which are explained by the slow-fast dynamics of the fast subsystems, and regions of very sharp peaks and valleys, which correspond to $\mathcal{S}_{I I I}$. (Note that the regions of constant phase can also be observed in Figure 24(b) and in Figure 25(b).) Surprisingly, the regions related to $\mathcal{S}_{I I I}$ exhibit a fractal geometry: zooming in simply reveals more and more self-similar patterns at smaller and smaller scales (Figure 26(b)). Owing to this property, $\mathcal{S}_{I I I}$ is interpreted as a phaseless set, characterized by arbitrarily high concentrations of isochrons.

This very high phase sensitivity near $\mathcal{S}_{I I I}$ is further demonstrated in Figure 27, which shows two trajectories initialized very close to one another but diverging at the beginning of the active bursting segment. (Note that, in some situations, this is also observed at the end of the active bursting segment.) This divergent behavior is captured in terms of the large phase difference (of the two red crosses in Figure 26(b), bottom). This can be explained in terms of the fast subsystems: it is induced by the effect of the slow manifold $\tilde{S}$, which separates the trajectories leaving $\tilde{Q}_{s}$ and converging toward $\tilde{\Gamma}_{s}$. A similar phenomenon was observed for 


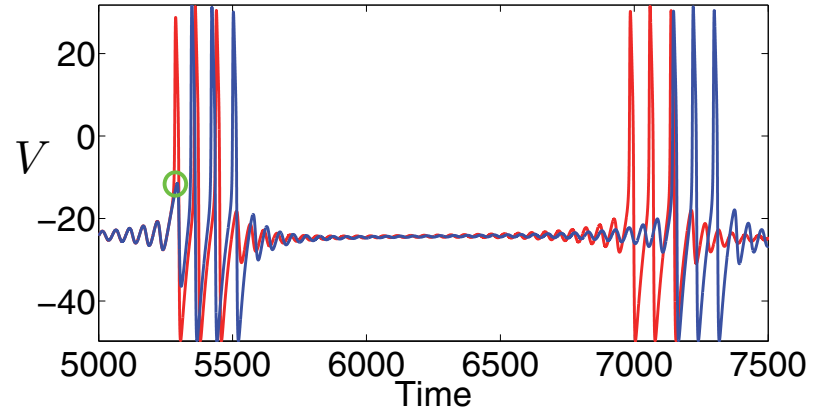

(a)

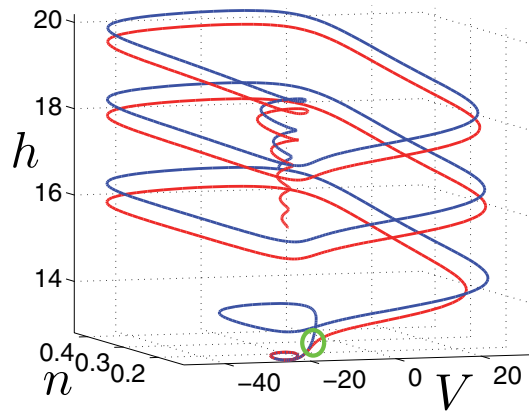

(b)

Figure 27. Two trajectories initialized very close to one another, but near $\mathcal{S}_{I I I}$. The trajectories diverge from one another at the beginning of the active bursting segment (green circle), after almost three revolutions along the limit cycle (at time $t \approx 5200$ ). This highlights the high sensitivity of the model. The initial conditions are $V=20, h=17$, and $n=0.30710505$ (blue curve) or $n=0.30710505+3 \times 10^{-7}$ (red curve). They correspond to the red crosses in Figure 26(b), bottom. In (b), the trajectories are shown over the time interval $t \in[5200,5700]$.

trajectories with initial conditions near $\mathcal{S}_{I I}$, but not as strongly. When the initial condition is near $\mathcal{S}_{I I}$, the trajectories diverge at the end of the burst, after less than one time period $T_{0}$. In contrast, the trajectories initialized near $\mathcal{S}_{I I I}$ wait more than one period before finally diverging (e.g., almost three time periods in Figure 27).

The properties of $\mathcal{S}_{I I I}$ - fractal geometry (see Figure 26) and high sensitivity to initial conditions (see Figure 27) - are reminiscent of the well-known chaotic features of particular bursting models [6]. More precisely, they suggest the existence of a chaotic saddle [44]. Because of these properties, the visualization of the two-dimensional isochrons proved too difficult, even with the adaptive grid approach in a small region of the state space.

7. Conclusion. We have investigated the full phase sensitivity of the well-known bursting Hindmarsh-Rose model. For that purpose, we used the Koopman operator framework and the Fourier averages method recently proposed in [31], associated with a computation on adaptive grids. For the first time, the two-dimensional isochrons of the full model have been computed, and their complex geometry has been described in detail. These results showed that singular perturbation theory cannot be used to obtain a complete and detailed description of the phase sensitivity of bursting models.

One of the main results of this study has been to highlight and describe a particular subset of the state space characterized by a high concentration of isochrons: the so-called almost phaseless set. The existence of the almost phaseless set sheds new light on various well-known phenomena. For instance, the addition of spikes and the early termination of the burst under particular perturbations is directly related to the crossing of the almost phaseless set. A similar set of very high phase sensitivity is also observed in an elliptic bursting model and is shown to exhibit fractal properties.

Our analysis also considered the phase response of the Hindmarsh-Rose model to noninfinitesimal impulsive perturbations. In particular, the effects of such perturbations - i.e., 
phase lag or phase advance - at different phases on the limit cycle are summarized in Figures 18 and 19. These results were also used to design optimal and efficient perturbations that trigger large phase shifts. In the case of elliptic bursting, however, the fact that the regions of high sensitivity are less localized and fractal implies that the response of the neuron is unreliable, especially when the perturbations push the trajectories into the set $\mathcal{S}_{I I I}$. This seems to complicate - or even prevent - the design of efficient perturbations for elliptic bursting models.

Future work. A straightforward extension of the present results and methodology is to study the effect and the efficiency of continuous perturbations, instead of impulsive perturbations. Moreover, the (noninfinitesimal) phase response function computed in this paper provides a global description of the bursting neuron model that is in line with phase-amplitude approaches recently developed in $[7,45]$. The phase response function might be used in this context to investigate the synchronization properties of (pulse-coupled) bursting neurons, and theoretical results could be obtained with simple approximations of this function. The framework could also be used to complement existing results on the effect of noise on bursting neuron models [23, 43]. In addition, phase response functions of different bursting models could be compared through the method proposed in [40], providing an alternative approach to classifying bursting models. Finally, a complete description of the two-dimensional isochrons is still missing in the case of elliptic bursting. The isochrons of other high-dimensional bursting neuron models (e.g., parabolic bursting) could also be computed, but their representation would be possible only in well-chosen three-dimensional cross sections of the state space.

\section{Appendix A. Simulation details.}

A.1. Refinement criteria. The key to the adaptive grid is to choose a good set of refinement criteria for when a given leaf cell is to be refined or split (i.e., given children). First, we define the distance function $d: \mathbb{S}^{1} \times \mathbb{S}^{1} \rightarrow[0, \pi]$ for two phase values on the circle by

$$
d\left(\theta_{1}, \theta_{2}\right):=\min _{k \in \mathbb{Z}}\left|\theta_{1}-\theta_{2}+k 2 \pi\right|,
$$

i.e., as the length of the smallest possible arc between them. Second, we denote the values of $\Theta$ computed at vertex $i$ by $\theta_{i}$. A given leaf cell is refined if and only if it neighbors a smaller cell that has just been refined (gradedness property) or it satisfies all three of the following refinement criteria:

1. The cell satisfies at least one of the two conditions:

(a) There exists one edge of the cell with vertices $i$ and $j$ such that $d\left(\theta_{i}, \theta_{j}\right)>$ $d \Theta_{\text {desired }}$, where the parameter $d \Theta_{\text {desired }}$ is the given maximum allowed value;

(b) $d\left(\theta_{c}, \theta_{m}\right)>e \Theta_{\text {desired }}$, where $\theta_{c}$ is the phase value at the center of the cell, $\theta_{m}$ is the average of the phases $\theta_{i}$ at the vertices (see the remark below), and the parameter $e \Theta_{\text {desired }}$ is the given maximum allowed "error" value.

Note that choosing $d \Theta_{\text {desired }}>\pi$ (resp., $e \Theta_{\text {desired }}>\pi$ ) is equivalent to ignoring condition (a) (resp., condition (b)).

2. There exists an edge with vertices $i$ and $j$ and a phase value $\theta \in C_{\text {desired }}$ such that $d\left(\theta_{i}, \theta\right)+d\left(\theta_{j}, \theta\right)=d\left(\theta_{i}, \theta_{j}\right)$-i.e., $\theta$ lies on the smallest arc between $\theta_{i}$ and $\theta_{j}$, where $C_{\text {desired }} \subseteq \mathbb{S}^{1}$ is a desired set of isochrons. In other words, the cell intersects a desired isochron. Note that choosing $C_{\text {desired }}=\mathbb{S}^{1}$ is equivalent to ignoring the criterion. 
3. $L<L_{\text {desired, }}$, where $L$ is the cell's level in the overall tree and $L_{\text {desired }}$ is the given maximum allowed level.

Subcriterion 1(b) was found to be necessary to capture certain sharp trenches or "negative peaks" in the phase function, which are not captured by subcriterion 1(a) when the phase difference between the vertices is small. On the other hand, subcriterion 1(b) alone is insufficient when the phase differences between the vertices are large, since then the averaging of more than two values on the circle leads to irrelevant values of $\theta_{m}$. Criterion 2 is used to selectively refine along certain isochrons, chosen a priori; it proves especially useful when computing in the full three-dimensional state space. Criterion 3 is necessary to avoid the unreasonably many if not infinite refinements that would otherwise occur near the (almost) phaseless set, where the phase differences and error values are inherently large.

The refinement algorithm is easy to use incrementally: if one finds that the specified values are slightly insufficient, one can decrement $d \Theta_{\text {desired }}$ and/or $e \Theta_{\text {desired }}$ and/or increment $L_{\text {desired }}$ and continue the refinement on the same data set. This is an added advantage of using adaptive grids.

A.2. Grid initialization. As mentioned in section 3.2, the initial number of grid cells in each direction determines the aspect ratio of the cells and therefore impacts the efficiency of the refinement process. Unlike the refinement parameters, this number cannot be modified later in the computation and must be carefully chosen.

A first rule to follow is that the phase differences between neighboring cells should be approximately the same in each direction. For instance, for the phase response computation of section 5 , the initial number of cells is much larger in the $\tilde{\theta}$ direction than in the $\alpha$ direction.

Secondly, the total number of initial grid cells should be large enough so as not to miss small scale variations in phase, for instance if they happen to be extremely localized. However, as long as some variation is detected initially, refinements near even the smallest features tend to propagate outward very rapidly, revealing any other features in the vicinity; this is one of the key benefits of the gradedness requirement.

A.3. Level set computation. For the purposes of visualizing the phase function and computing its level sets, i.e., the isochrons, we have first generated a triangle mesh from the quadtree (resp., octree) grid. Rather than attempting to explicitly constrain triangles (tetrahedrons) to the grid cells, we opted for the well-known Delaunay triangulation (resp., tetrahedralization) method. Specifically, we used the MATLAB function "delaunay" to generate the triangles (resp., tetrahedrons). All of our plots of the phase function $\Theta$ and the gradient $\nabla \Theta$ use these triangles and the function "trisurf," usually with shading based on linear interpolation.

Once one defines a triangular (resp., tetrahedral) mesh from the quadtree (resp., octree) grid, the definition of a given level set is relatively straightforward, via the marching triangles (resp., tetrahedrons) algorithm. There are two main parts of the algorithm: (a) determining where a given level set intersects a given edge of the triangular (resp., tetrahedral) mesh, if anywhere, via inverse linear interpolation, and (b) correctly connecting the resulting level set points to form line segments (resp., triangular faces). Note that although one could extend a contour algorithm such as "marching squares (resp., cubes)" to work on the quadtree 
(resp., octree) grid directly, we found the "marching triangles (resp., tetrahedrons)" framework more straightforward.

In the octree case, the resulting level sets were smoothed using the MATLAB function "smoothpatch" created by Dirk-Jan Kroon. This was done to correct for the well-known problem of artificial "bumps" inherent to the marching tetrahedrons algorithm that are caused by the slightly higher interpolation error along the longest tetrahedron edges in a given cell.

A caveat to the level set computation, which also affects refinement criteria 1(a) and 1(b), is that the values lie in $\mathbb{S}^{1}$ rather than in $\mathbb{R}$. For instance, an interval such as $[-\pi+0.1, \pi-0.1]$ includes the value 0 if it lies in $\mathbb{R}$, but does not include the value 0 if it lies in $\mathbb{S}$ and has a length equal to 0.2 . In general an edge of the mesh effectively intersects a level set only if the values of $\Theta$ at its endpoints form an interval of length less than $\pi$ that contains the level set value of interest. This condition is stated mathematically in refinement criterion 2. Though seemingly simple, the need for this condition actually makes the procedure for connecting the level set points ((b) above) significantly more complicated, and renders existing implementations useless. As such, it was necessary to implement custom versions of both the marching triangles algorithm and the marching tetrahedrons algorithm that consider values not only in $\mathbb{R}$ but also in $\mathbb{S}^{1}$.

Even with these phase-friendly implementations, it is inherently impossible to obtain numerically a level set on a given triangle (or tetrahedron) if this triangle (or tetrahedron) intersects too many other level sets. In the presence of a high concentration of level sets, an edge of the mesh is likely to span a phase interval of length larger than $\pi$. As a consequence, the contour algorithm, which assumes by default the interval length to be less than $\pi$, might not capture some level sets, leaving holes in the triangular surfaces (in three dimensions). This was observed near the almost phaseless set and, more so, near the phaseless set where the concentration of level sets is arbitrarily large. Also, as an attempt to reduce the phase interval spanned by a cell below $\pi$, increasing the resolution merely yields more smaller holes in the vicinity of the phaseless set (see Figure 5).

Because of that issue, the three-dimensional representation of the isochrons of Figures 1417 were computed with values considered in $\mathbb{R}$ instead of $\mathbb{S}^{1}$ (standard marching tetrahedrons algorithm). Since the overall ranges of values spanned by the isochrons $\left(\left[-\frac{3 \pi}{36}, \frac{\pi}{36}\right],\left[-\frac{5 \pi}{18},-\frac{3 \pi}{18}\right]\right.$, $\left[-\frac{4 \pi}{6},-\frac{3 \pi}{6}\right]$, and $\left[\frac{10 \pi}{36}, \frac{13 \pi}{36}\right]$, respectively) have lengths significantly smaller than $\pi$ and do not include the value $\pi=-\pi$, this in fact does yield the same result, just without the holes.

A.4. Gradient. The norm of the gradient $\|\nabla \Theta\|$ was computed in planar cross sections of the state space, and the e component of the gradient was used in the computation of the PRE $Z_{e}$ for $\alpha=0$, according to (2.7).

The gradient $\nabla \Theta$ is approximated by using the Delaunay triangulation. We first compute the gradient $\nabla \Theta$ on each triangle, taking into account that the vertex values $\theta_{i}, \theta_{j}$, and $\theta_{k}$ lie in $\mathbb{S}^{1}$ not $\mathbb{R}$. This is done by shifting all three of these values (by multiples of $2 \pi$ ) to each of $\left[\theta_{i}, \theta_{i}+2 \pi\right),\left[\theta_{j}, \theta_{j}+2 \pi\right)$, and $\left[\theta_{k}, \theta_{k}+2 \pi\right)$. Each of these three intervals yields a unique planar version of $\Theta$ and thus a trivially defined gradient $\nabla \Theta$. We simply use the version of $\nabla \Theta$ with the smallest norm $\|\nabla \Theta\|$. The gradient $\nabla \Theta$ is then computed at each vertex as the average of the values at the neighboring triangles weighted with the inverses of the distances to the triangle centers. 


\section{Appendix B. Simulation parameters.}

\section{B.1. HR model.}

Numerical integration. We used the MATLAB function "ode45" with a relative error of $10^{-4}$ for the full system and with a relative error of $10^{-6}$ and an absolute error $10^{-50}$ for the fast subsystem.

Fourier averages. We used the function $f(V, n, h)=n$. For the full subsystem, we computed the truncated Fourier averages (3.2) with the parameters $T=2000$ and $N=1$ (with $\omega_{0} \approx$ $0.0145854, T_{0} \approx 430.786$ ). For the fast subsystem, we computed the (nontruncated) Fourier averages (3.1) with $T=500$ and $\omega_{0}=0.44667$.

Adaptive grids. The parameters are given in Table 1.

Table 1

Adaptive grid parameters for the HR model.

\begin{tabular}{cccccc}
\hline Figure \# & Initial grid & $\begin{array}{c}\text { Criterion 1(a) } \\
d \Theta_{\text {desired }}\end{array}$ & $\begin{array}{c}\text { Criterion 1(b) } \\
e \Theta_{\text {desired }}\end{array}$ & $\begin{array}{c}\text { Criterion 2 } \\
C_{\text {desired }}\end{array}$ & $\begin{array}{c}\text { Criterion 3 } \\
L_{\text {desired }}\end{array}$ \\
\hline $2,3,7,8,9,11$ & $40 \times 40$ & 1 & 0.1 & $\mathbb{S}^{1}$ & 3 \\
10 & $50 \times 50$ & 0 & 0 & $\subset \mathbb{S}^{1}$ & 5 \\
$5,14-17$ & $5 \times 5 \times 5$ & 0 & 0 & $\subset \mathbb{S}^{1}$ & 4 \\
$18-21$ & $100 \times 12$ & 0.25 & $>\pi$ & $\mathbb{S}^{1}$ & 5 \\
\hline
\end{tabular}

\section{B.2. Elliptic bursting model.}

Numerical integration. We used the MATLAB function "ode45" with a relative error of $10^{-6}$ and an absolute error of $10^{-300}$ for the full system and with a relative error of $10^{-6}$ and an absolute error of $10^{-50}$ for the fast subsystem.

Fourier averages. For the full system, we used the function $f(V, n, h)=h$, and we computed the truncated Fourier averages (3.2) with the parameters $T=8500$ and $N=1$ (with $\omega_{0} \approx$ $\left.0.003701168, T_{0} \approx 1697.62\right)$. For the fast subsystem, we used the function $f(V, n, h)=n$ and we computed the (nontruncated) Fourier averages (3.1) with $T=5000$ and $\omega_{0}=0.07919778$.

Adaptive grids. The parameters are given in Table 2.

Table 2

Adaptive grid parameters for the elliptic bursting model.

\begin{tabular}{cccccc}
\hline Figure \# & Initial grid & $\begin{array}{c}\text { Criterion 1(a) } \\
d \Theta_{\text {desired }}\end{array}$ & $\begin{array}{c}\text { Criterion 1(b) } \\
e \Theta_{\text {desired }}\end{array}$ & $\begin{array}{c}\text { Criterion 2 } \\
C_{\text {desired }}\end{array}$ & $\begin{array}{c}\text { Criterion 3 } \\
L_{\text {desired }}\end{array}$ \\
\hline $24(\mathrm{a}), 25(\mathrm{a})$ & $40 \times 40$ & 0.2 & 0.02 & $\mathbb{S}^{1}$ & 3 \\
$24(\mathrm{~b}), 25(\mathrm{~b})$ & $100 \times 100$ & 0.1 & 0.01 & $\mathbb{S}^{1}$ & 2 \\
\hline
\end{tabular}

\section{REFERENCES}

[1] R. Bertram, M. J. Butte, T. Kiemel, and A. Sherman, Topological and phenomenological classification of bursting oscillations, Bull. Math. Biol., 57 (1995), pp. 413-439.

[2] A. BorisyUK AND J. Rinzel, Understanding neuronal dynamics by geometrical dissection of minimal models, in Methods and Models in Neurophysics, Proceedings of Les Houches Summer School, Elsevier, New York, 2005, pp. 19-72. 
[3] E. Brown, J. Moenlis, And P. Holmes, On the phase reduction and response dynamics of neural oscillator populations, Neural Comput., 16 (2004), pp. 673-715.

[4] M. Budišić, R. Mohr, And I. Mezić, Applied Koopmanism, Chaos, 22 (2012), 047510.

[5] R. J. Butera, J. Rinzel, And J. C. Smith, Models of respiratory rhythm generation in the pre-Bötzinger complex. I. Bursting pacemaker neurons, J. Neurophysiol., 82 (1999), pp. 382-397.

[6] C. C. Canavier, J. W. Clark, and J. H. Byrne, Routes to chaos in a model of a bursting neuron, Biophys. J., 57 (1990), pp. 1245-1251.

[7] O. Castejón, A. Guillamon, and G. Huguet, Phase-amplitude response functions for transient-state stimuli, J. Math. Neurosci., 3 (2013), pp. 1-26.

[8] T. R. Chay And J. KeIZer, Minimal model for membrane oscillations in the pancreatic beta-cell, Biophys. J., 42 (1983), pp. 181-189.

[9] D. C. CoOper, The significance of action potential bursting in the brain reward circuit, Neurochem. Internat., 41 (2002), pp. 333-340.

[10] S. S. Demir, R. J. Butera, A. A. DeFranceschi, J. W. Clark, and J. H. Byrne, Phase sensitivity and entrainment in a modeled bursting neuron, Biophys. J., 72 (1997), pp. 579-594.

[11] G. B. Ermentrout And N. Kopell, Oscillator death in systems of coupled neural oscillators, SIAM J. Appl. Math., 50 (1990), pp. 125-146.

[12] G. B. Ermentrout And D. H. Terman, Mathematical Foundations of Neuroscience, Springer, New York, 2010.

[13] N. Fenichel, Persistence and smoothness of invariant manifolds for flows, Indiana Univ. Math. J., 21 (1971), pp. 193-226.

[14] N. Fenichel, Asymptotic stability with rate conditions, Indiana Univ. Math. J., 23 (1973), pp. 1109-1137.

[15] A. Franci, G. Drion, And R. Sepulchre, Modeling the modulation of neuronal bursting: A singularity theory approach, SIAM Appl. Dyn. Syst., to appear; http://arXiv.org/abs/1305.7364, 2013.

[16] M. Golubitsky, K. Josic, And T. J. Kaper, An unfolding theory approach to bursting in fast-slow systems, in Global Analysis of Dynamical Systems, Institute of Physics Publishing, London, 2001, pp. $277-308$.

[17] W. Govaerts And B. Sautois, Computation of the phase response curve: A direct numerical approach, Neural Comput., 18 (2006), pp. 817-847.

[18] J. Guckenheimer, Isochrons and phaseless sets, J. Math. Biol., 1 (1975), pp. 259-273.

[19] J. GuCKenheimer And C. Kuehn, Computing slow manifolds of saddle type, SIAM J. Appl. Dyn. Syst., 8 (2009), pp. 854-879.

[20] A. Guillamon And G. Huguet, A computational and geometric approach to phase resetting curves and surfaces, SIAM J. Appl. Dyn. Syst., 8 (2009), pp. 1005-1042.

[21] C. Hammond, H. Bergman, And P. Brown, Pathological synchronization in Parkinson's disease: Networks, models and treatments, Trends Neurosci., 30 (2007), pp. 357-364.

[22] J. L. Hindmarsh and R. M. Rose, A model of neuronal bursting using three coupled first order differential equations, Proc. Roy. Soc. London Ser. B Biol. Sci., 221 (1984), pp. 87-102.

[23] P. Hitczenko And G. S. Medvedev, Bursting oscillations induced by small noise, SIAM J. Appl. Math., 69 (2009), pp. 1359-1392.

[24] G. Huguet And R. DE la Llave, Computation of limit cycles and their isochrons: Fast algorithms and their convergence, SIAM J. Appl. Dyn. Syst., 12 (2013), pp. 1763-1802.

[25] E. M. Izhikevich, Neural excitability, spiking and bursting, Internat. J. Bifur. Chaos Appl. Sci. Engrg., 10 (2000), pp. 1171-1266.

[26] E. M. IzhIKEVICH, Synchronization of elliptic bursters, SIAM Rev., 43 (2001), pp. 315-344.

[27] E. M. IzHikevich, Dynamical Systems in Neuroscience: The Geometry of Excitability and Bursting, MIT Press, Cambridge, MA, 2007.

[28] J. Keener and J. Sneyd, Mathematical Physiology, Springer-Verlag, New York, 1998.

[29] Y. Kuramoto, Chemical Oscillations, Waves, and Turbulence, Springer-Verlag, New York, Berlin, 1984.

[30] I. G. Malkin, The Methods of Lyapunov and Poincaré in the Theory of Nonlinear Oscillations, Gostekhizdat, Moscow, Leningrad, 1949.

[31] A. Mauroy And I. Mezić, On the use of Fourier averages to compute the global isochrons of (quasi)periodic dynamics, Chaos, 22 (2012), 033112.

[32] I. Mezić And A. Banaszuk, Comparison of systems with complex behavior, Phys. D, 197 (2004), pp. 101133. 
[33] V. Novičenko and K. Pyragas, Computation of phase response curves via a direct method adapted to infinitesimal perturbations, Nonlinear Dynamics, 67 (2012), pp. 517-526.

[34] H. M. Osinga And J. Moehlis, Continuation-based computation of global isochrons, SIAM J. Appl. Dyn. Syst., 9 (2010), pp. 1201-1228.

[35] H. M. Osinga, A. Sherman, and K. Tsaneva-Atanasova, Cross-currents between biology and mathematics: The codimension of pseudo-plateau bursting, Discrete Contin. Dyn. Systems, 32 (2012), pp. 2853-2877.

[36] J. RinzeL, A formal classification of bursting mechanisms in excitable systems, in Proceedings of the International Congress of Mathematicians, Springer, New York, 1986, vol. 1, pp. 1578-1593.

[37] J. Rinzel And G. B. Ermentrout, Analysis of neural excitability, in Methods of Neuronal Modeling, C. Koch and I. Segev, eds., MIT Press, Cambridge, MA, 1998, pp. 251-291.

[38] J. Rinzel And Y. S. LeE, Dissection of a model for neuronal parabolic bursting, J. Math. Biol., 25 (1987), pp. 653-675.

[39] J. E. Rubin, C. C. McIntyre, R. S. Turner, and T. Wichmann, Basal ganglia activity patterns in Parkinsonism and computational modeling of their downstream effects, European J. Neurosci., 36 (2012), pp. 2213-2228.

[40] P. Sacré And R. Sepulchre, Sensitivity analysis of oscillator models in the space of phase response curves: Oscillators as open systems, IEEE Control Systems Mag., 2014, to appear; http://arxiv.org/ abs/1206.4144.

[41] W. E. Sherwood And J. Guckenheimer, Dissecting the phase response of a model bursting neuron, SIAM J. Appl. Dyn. Syst., 9 (2010), pp. 659-703.

[42] A. Shilnikov and M. Kolomiets, Methods of the qualitative theory for the Hindmarsh-Rose model: A case study. A tutorial, Internat. J. Bifur. Chaos Appl. Sci. Engrg., 18 (2008), pp. 2141-2168.

[43] J. Su, J. Rubin, And D. Terman, Effects of noise on elliptic bursters, Nonlinearity, 17 (2004), pp. 133157.

[44] T. TÉL And M. Gruiz, Chaotic Dynamics: An Introduction Based on Classical Mechanics, Cambridge University Press, Cambridge, UK, 2006.

[45] K. C. A. Wedgwood, K. K. Lin, R. Thul, and S. Coombes, Phase-amplitude descriptions of neural oscillator models, J. Math. Neurosci., 3 (2013), pp. 1-22.

[46] A. Winfree, The Geometry of Biological Time, 2nd ed., Springer-Verlag, New York, Berlin, 2001.

[47] A. T. Winfree, Patterns of phase compromise in biological cycles, J. Math. Biol., 1 (1974), pp. 73-95. 